\title{
An Experimental Evaluation on the awareness of "Pradhan Mantri Jan Aryogya Yojna - Ayushmaan Bharat" (PMJAY-AB) Scheme among residents of Moradabad, Uttar Pradesh
}

\author{
Vipin Jain, Vivek D. Singh, Khushboo
}

\begin{abstract}
Background: One of the most ambitious health insurance programme of the world was launched in September 2018 i.e. Pradhan Mantri Jan Arogya Yojana (PMJAY) popularly known as Ayushmaan Bharat. The programme aims to help the 100 million poor families of India to get the benefits of medical services for their health and take the treatment of disease which they cannot afford. This gives a chance to Shri Narendera Modi's government to transform the health care sector of India. This paper discuss about the awareness of PMJAY-AB programme among the residents of Moradabad, Uttar Pradesh.

Material \& Methods: It empirical study done on the residents of Moradabad region in Uttar Pradesh by Around 150 samples were collected in which 130 samples were found correct, as they were completely filled and used as a data for analysis. This topic was taken for study to assess people awareness regarding government schemes in health care sector which is associated with hospital and health care management by applying Chi-Square Test, Analysis of Variance (ANOVA) and Pearson's Correlation Test. The period of study was of 4 months i.e. from February 2019 to May 2019. The cluster convenient sampling method was chosen for the study. For the collection of data the Moradabad was divided into three parts:-
\end{abstract}

1. MDA- near Vivekanand Hospital,

2. Majhola, Mansarover \& Budhi Vihar - near Sai Hospital and

\section{Pakbara-near TMU Hospital}

Results \& Conclusion: The study concludes that the demographic factors have some relationship, effect and correlation on PMJAY - AB awareness at Moradabad. These demographic factors gender, age, marital status, religions, education qualification, occupations \& income status were tested with resident's awareness status of PMJAY-AB by conducting descriptive analysis, chi-square test (Relationship), analysis of variance \& post hoc (Effect) and correlation (Association). RELATIONSHIP: It is hereby concluded from the results obtained by applying chi-square test (cross tabulation) that except demographic variable Age groups no other variable has a significant relationship with the resident's awareness of PMJAY - AB at Moradabad. EFFECT: It is hereby concluded from the results obtained by conducting ANOVA that age groups, education level and income-socio-economic level play

Revised Manuscript Received on September 15, 2019.

* Correspondence Author

Professor Vipin Jain, Principal/Director TMIMT, Teerthanker Mahaveer University, Moradabad. UP.

Mr. Vivek D. Singh, Assistant Professor TMIMT, Teerthanker Mahaveer University, Moradabad UP.

Dr. Khushboo, Practitioner (BDS), Moradabad, UP. role in effecting the resident's awareness regarding $P M J A Y-A B$ at Moradabad. It is also concluded that very old people (above 60 yrs) have highest awareness of PMJAY-AB and young generation (20-30 yrs) have the lowest awareness regarding this government scheme. Further residents between age bands 31-60 yrs have moderate awareness in the Moradabad City. When it comes to education level awareness about PMJAY-AB scheme is least among under graduates and there is no difference between the Awareness of PMJAY-AB among graduates and post graduates in Moradabad. Other demographic variable does not have any effect on awareness of this government scheme in Moradabad. ASSOCIATION: It is hereby concluded from the results obtained after applying Pearson_Correlation(r) test that except age groups no other variable (education qualification and income) make any association with awareness level of PMJAYAB scheme at Moradabad.

Keywords : Pradhan Mantri Jan Arogya Yajna (PMJAY), Ayushmaan Bharat (AB), Health Insurance, Health Care Sector, Universal Health Care (UHC)

\section{RESEARCH OBJECTIVES}

General objective of the study is to understand the awareness of PMJAY scheme among the residents of Moradabad city on the basis of gender, marital status, religion, education qualifications, occupation and income-socio-economic status. The objective can be as follows:

1) To assess the relationship between demographic factors and awareness about PMJAY-AB.

2) To find the effect of demographic factors on the level of awareness about PMJAY-AB.

3) To find the impact of demographic factors on the level of awareness about PMJAY-AB.

\section{INTRODUCTION}

The most critical concern of life is health. Health is very important to those persons whose earning is totally dependent on the manual works i.e. they do all the work by their body parts due to which their body becomes the prime assets for them. Health is also very important for others because the productivity of their work and quality of the life is totally dependent on their wellness of the body. Health of population is an asset for any society or nation in the world; hence it is called as human capital of the nation or society. 


\section{An Experimental Evaluation on the awareness of "Pradhan Mantri Jan Aryogya Yojna - Ayushmaan Bharat" (PMJAY-AB) Scheme among residents of Moradabad, Uttar Pradesh}

So the good health creates a positive impact on the values and capabilities of human. On the other hands any disease, illness or an accident not only causes pain, discomfort but also financial losses to the family of sufferer. The costs of living of families are increases when any member of family is suffering from bad health. The evaluation of organized medical and health care system was started about 100 years ago. The evaluation of the organized health care has increases the reach and combating diseases and cure of disabilities in all over the world due to which longevity and prosperity has been increased in manifolds. But this creates a massive demand in health care sector due to which the cost of health care is increasing day by day and it become un affordable for a common man of the society. So it is clear from above facts that health is most valuable asset for any individual, so to fight against the disease, an individual always requires the proper medical facilities. Today the costs of these facilities are increasing day by day. So individuals are need of financial protection against the health risks.

Now a days the concept of good health changes from not only curing the disease or injury but towards preventive and rehabilitative care of individuals. But in India stakeholders are fully focused on curing of disease and injuries. The market of preventive and rehabilitative care is still under consideration and various stakeholders are still exploring this market. Around 175 million poor children worldwide are affected from under nutrition and related conditions out of which 3.45 million dies due to under nutrition and related conditions. Around 1.2 billion adults are overweight and 345 million adults are clinically obese. There is a change in living patterns lot of peoples are migrating from villages to modern cities and migration of labour of agriculture (farming) to industries or service sector situated in developed areas are also creating risk to health. So health risk is not only affecting poor but also affecting the rich segment of the society.

Due to the above scenario new market in health care insurance had been emerged after the LPG (Liberalization, Privatization and Globalization) adopted by Mr. Man Mohan Singh government. This strategy of government not only improves the spending power of middle class but also open the door to global health insurance companies. Total policies sold in year 2001 were 7.54 million and increased to 10.04 million policies by year 2004. The market had been expanded by manifolds during first decade of this century. To provide proper customer satisfaction and control on the working of health care sector companies the new body was set up in name of Insurance Regulatory and Development Authority of India (IRDA) on January 1, 2007. The PHD Chamber of Commerce and Industry expected the growth of health insurance sector to $\$ 5.72$ billion by 2010 . Still this sector was not able to provide full justification to the population of the country in health care services which leads government to think again and take some remedial measures to strengthen the health insurance sector. In year $2014 \mathrm{Mr}$. Narender Damodardas Modi became prime minister of India and in September 2018 he announced dream project for health care insurance naming "Pradhan Mantri Jan Aryogya Yojna -
Ayushmaan Bharat" (PMJAY-AB). One of the most ambitious health insurance programme of the world. The programme aims to help the 100 million poor families of India to get the benefits of medical services for their health and take the treatment of disease which they cannot afford. This gives a chance to Shri Narendera Modi's government to transform the health care sector of India. This paper discuss about the awareness of PMJAY programme among the residents of Moradabad, Uttar Pradesh.

Since India is developing country where a large number of population is below poverty line, proper health and maintain hygiene are not up to the mark. The mortality rate of pregnant women, infant die during child birth and a huge amount of people die due to disease such as Tuberculosis, HIV/AIDS, Hepatitis, Cancer, Oral cancer, etc. Moreover the costs and expenses associated with health care facilities are shooting up like anything at private health care centres, hospitals, super speciality hospitals, etc. which are out of reach for the common people, but are now essential, as Government bodies and structure does not provide such health care, and if they dose, are very scarce. As health care is a social need, the social bodies like NGO, trusts, associations started some hospitals with reasonable price structure. But today such health care hospitals are now charging premium and are not catering services for the poor and needy. As population in India is increasing manifold it's the Government which need to take some decision on social welfare for poor and needy. On 14th April 2018 government of India launched a health insurance programme scheme named as "Pradhan Mantri Jan Aryogya Yojna - Ayushmaan Bharat" (PMJAY-AB), it is also known as "Ayushman Bharat" or National Health Protection Scheme which consist of two major parameters :-

- National Health Protection Mission - to provide cashless treatment to patients and

- Wellness Centres - to provide primary care to the patients.

10 Crore poor people (below poverty line) and vulnerable families are covered providing coverage up to 5 Lakh rupees per family per year with secondary and tertiary care hospital facilities.

Awareness about health insurance scheme include awareness in general population, which include both urban as well as rural about its availability, eligibility of coverage, benefit of the scheme costs, usefulness and problems occurred while getting claims.

In India, there is lack of awareness of any scheme launched by government especially in the rural areas, hence this study was undertaken to determine the awareness among residents of Moradabad, Uttar Pradesh, India. 


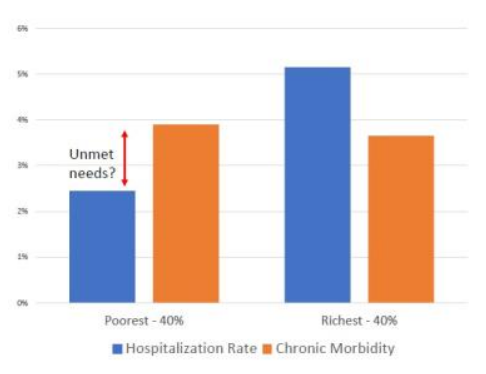

Source: Chronic morbidity: IHDS 2011-2012

Hospitalization Rate: NSSO 71st (excludes childbirth)

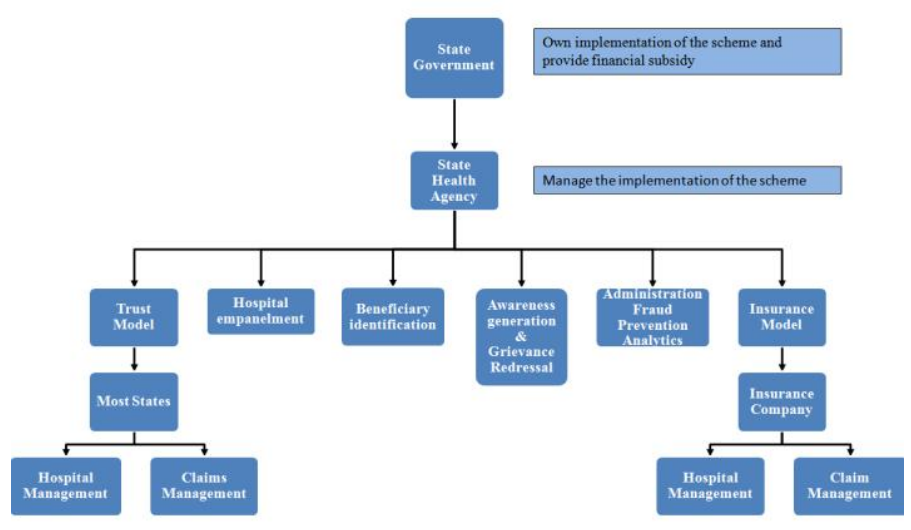

Source: https://www.pmjay.gov.in/

\section{BACKGROUND OF STUdY}

India is the fastest growing economy of the world. According to the report (2011) ${ }^{[1]}$ of World Health Organization (WHO) total expenditure incurred by India was 3.9 percent of GDP which was lowest among the member of BRICS (Brazil, Russia, China, and South Africa). The amount spent from personal account was 82 percent, around 9 percent from employee account and remaining from health insurance account i.e. 5 to 10 percent. ${ }^{[2]}$ According to Confederation of Indian Industry (CII) health care industry of India requires US dollar 50 billion every year for next 20 years to meet the demand created by increase in the purchasing power of middle class, increase in the number of insurance policies, increase in the life style diseases like cancer, diabetes, heart $\&$ respiratory diseases etc. ${ }^{[3]}$ In the survey carried out in the year 2014 by National Sample Survey office (NSSO), it was found out that more than $80 \%$ of Indians are not connected under any health insurance plan and only $18 \%$ (government funded $12 \%$ ) of urban population and $14 \%$ (government funded $13 \%$ ) of the rural was covered under any form of health insurance. ${ }^{[4]}$ To provide the financial supports to the poor families the government of India introduced Community health insurance (CHI) as part of National Health Mission. This study examines improvement in access to various hospital cares for the poor segment of society by using the dimensions of $\mathrm{CHI}$. ${ }^{[5]}$ this study shows that government health insurance scheme has less penetration in poor segment of the society. This study reveals that insurance companies were able provide customer satisfaction in various fields like delay or rejection in insurance claim, unfairness of selective health insurance, high insurance premium, difficulties in finding specialist physicians, hospitals were not able provide qualities in patient care. So there was need of starting new scheme that caters to the poor segment of society. ${ }^{[6]}$ This study reveals that more than $80 \%$ expenditure in health care is borne from the families own earning or savings, only 3 percent of population is benefited chronic India has given approvals to various Community Health enth, local NGOs (acting as insurer and care centre), second model was local NGO (acting as insurer only), third model was local NGO (nor as insurer and care provider). These models were failed to provide health care to poor segment of society. [7] This study reveals that the new term is used generally in developing countries for the insurance of poor segment of society is 'microfinance'. In this study researcher highlighted that most of the poor families are risk averse and they can not want extra expenditure to cover the risk. Families where more individuals are suffering from disease are taking benefits from these schemes. Generally the main perception in the society is not to go for such type of insurance as they are just wastage due lack of education. The premium is very high for them. ${ }^{[8]}$

\section{RESEARCH Methodology:}

It empirical study done on the residents of Moradabad region in Uttar Pradesh. Around 150 samples were collected in which 130 samples were found correct, as they were completely filled and used as a data for analysis. This topic was taken for study to assess people awareness regarding government schemes in health care sector which is associated with hospital and health care management. The period of study was of 4 months i.e. from February 2019 to May 2019. The cluster convenient sampling method was chosen for the study. For the collection of data the Moradabad was divided into three parts:-

1. MDA- near Vivekanand Hospital,

2. Majhola, Mansarover \& Budhi Vihar - near Sai Hospital and

\section{Pakbara - near TMU Hospital}

As per details available on website of Nagar Nigam Moradabad, the Moradabad is divided into 70 wards and above three areas of study covers the 9 wards of Moradabad. The total population of Moradabad city as per provisional report of Census India was 8.9 lakh out of which 4.6 lakh were male and 4.3 lakh were female. The data was collected through self-designed questionnaire by interview technique. The information was collected from various age group male and female, married, unmarried, illiterates, literates, Government servants, private job holders, housewives, businessmen and students.

\section{STudy Tool:}

Information was collected by using self designed semi-structured simple questionnaire regarding health insurance scheme by interview technique.

The following variables with 4 parts were collected:- 
An Experimental Evaluation on the awareness of "Pradhan Mantri Jan Aryogya Yojna - Ayushmaan Bharat" (PMJAY-AB) Scheme among residents of Moradabad, Uttar Pradesh

1. Information related to demographic factors like resident, age, gender, marital status, religion, education, occupation, and monthly income etc.

2. General awareness regarding "Pradhan Mantra Jan Arogya Yojna, - Ayushman Bharat" which include general information about the scheme and Health insurance covers treatments.

3. It includes questions related to the health insurance like status of eligibility under this scheme, status of registered name and related data.

4. It Includes questions related awareness about health insurance sector for example: type of scheme, availability of facility, list of treatment coverage under scheme, maximum limit of insured amount (in Rs) covered by the scheme, list of disease to be treated and amount of premium to be paid for the scheme.

\section{STATistical ANALYSIS:}

Data was entered in Microsoft Excel and analyzed using Statistical Package for Social Sciences (SPSS) version 20. Descriptive statistical measures like percentage, mean, and standard deviation were calculated. An inferential statistical measure like Chi Square Test, ANOVA and Correlation was applied to analyse relationship and variance differences to understand the awareness propositions among the residents of Moradabad City and were interpreted to be statistically significant at 0.05 levels.

\section{RESUlt AND ANALYSIS:}

\section{Classification of observations and data in Excel and SPSS}

20:

Among 130 people interviewed, which constituted by -

1. Gender (male and female denoted by 1 and 2 respectively),

2. Age Groups (classified into 4 parts 20-30yrs, 30-40yrs, 40-50yrs and above 50yrs denoted by 1,2, 3,4 and 5 respectively),

3. Marital Status (unmarried, married and widow denoted by 1, 2 and 3 respectively),

4. Religion (Hindu, Muslim and Jain denoted by 1, 2 and 3 respectively),

5. Education Qualification (pre-graduation, graduation and post-graduation denoted by $1,2,3$ and 4),

6. Occupation (housewife, student, business and services denoted by 1, 2, 3 and 4 respectively) and

7. Income (very low, low, average, high and very high denoted by 1, 2, 3, 4 and 5 respectively)

8. PMJAY - AB Awareness Category (very low, low, average, high and very high denoted by $1,2,3,4$ and 5 respectively)

\section{A.DESCRIPTIVE ANALYSIS Frequency Tables:}

1. Gender

\begin{tabular}{|l|l|l|l|l|l|}
\hline \multicolumn{2}{|c|}{} & $\begin{array}{l}\text { Frequ } \\
\text { ency }\end{array}$ & $\%$ & $\begin{array}{l}\text { Val } \\
\%\end{array}$ & $\begin{array}{l}\text { Cum } \\
\%\end{array}$ \\
\hline \multirow{2}{*}{$\begin{array}{l}\text { Valu } \\
\text { e }\end{array}$} & Male & 75 & 57.7 & 57.7 & 57.7 \\
\cline { 2 - 6 } & Female & 55 & 42.3 & 42.3 & 100.0 \\
\cline { 2 - 6 } & Total & 130 & 100.0 & 100.0 & \\
\hline
\end{tabular}

Table: 1

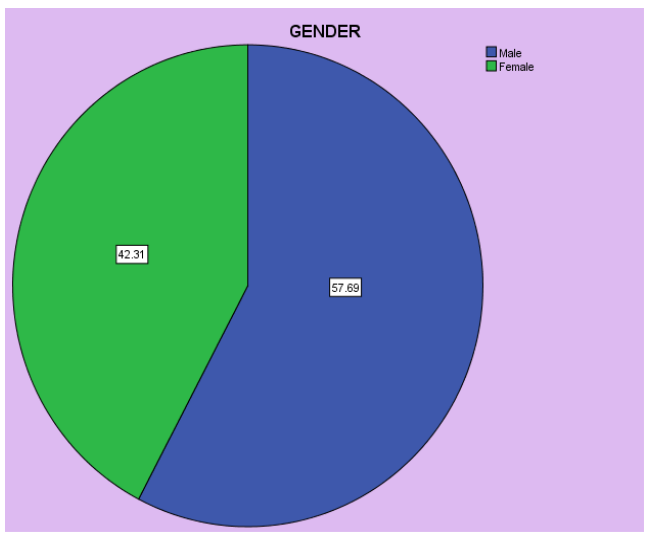

Figure: 1

2. Age Groups

\begin{tabular}{|c|c|c|c|c|c|}
\hline \multicolumn{2}{|c|}{ Age Groups } & $\begin{array}{c}\text { Freque } \\
\text { ncy }\end{array}$ & $\%$ & Val \% & $\begin{array}{c}\text { Cum } \\
\%\end{array}$ \\
\hline Value & $\begin{array}{c}\text { Young_Genera } \\
\text { tion }\end{array}$ & 17 & 13.1 & 13.1 & 13.1 \\
\hline \multirow{2}{*}{} & $\begin{array}{c}\text { Adult_Generatio } \\
\text { n1st }\end{array}$ & 56 & 43.1 & 43.1 & 56.2 \\
\cline { 2 - 6 } & $\begin{array}{c}\text { Adult_Generatio } \\
\text { n2nd }\end{array}$ & 37 & 28.5 & 28.5 & 84.6 \\
\cline { 2 - 6 } & $\begin{array}{c}\text { Old_BabyBoome } \\
\text { rs }\end{array}$ & 16 & 12.3 & 12.3 & 96.9 \\
\cline { 2 - 6 } & $\begin{array}{c}\text { VeryOld_BabyB } \\
\text { oomers }\end{array}$ & 4 & 3.1 & 3.1 & 100.0 \\
\cline { 2 - 6 } & Total & 130 & 100.0 & 100.0 & \\
\hline
\end{tabular}

Table: 2

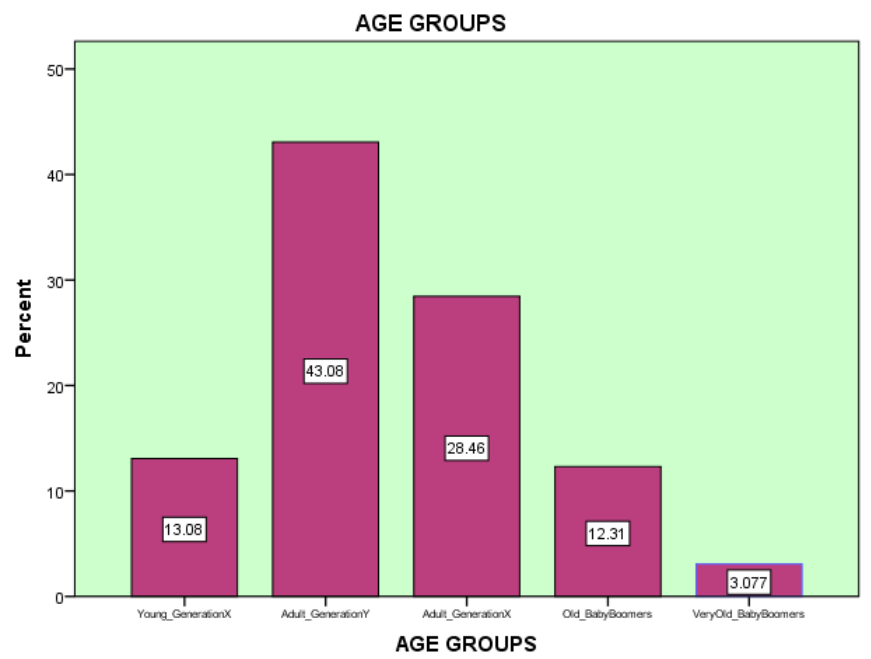

Figure: 2 
3. Marital Status

\begin{tabular}{|l|l|l|l|l|l|}
\hline \multicolumn{1}{|l|}{ Marital Status } & Frequency & $\%$ & Val \% & Cum \% \\
\hline \multirow{4}{*}{ Value } & Unmarried & 83 & 63.8 & 63.8 & 63.8 \\
\cline { 2 - 6 } & Married & 45 & 34.6 & 34.6 & 98.5 \\
\cline { 2 - 6 } & Widow & 2 & 1.5 & 1.5 & 100.0 \\
\cline { 2 - 6 } & Total & 130 & 100.0 & 100.0 & \\
\hline
\end{tabular}

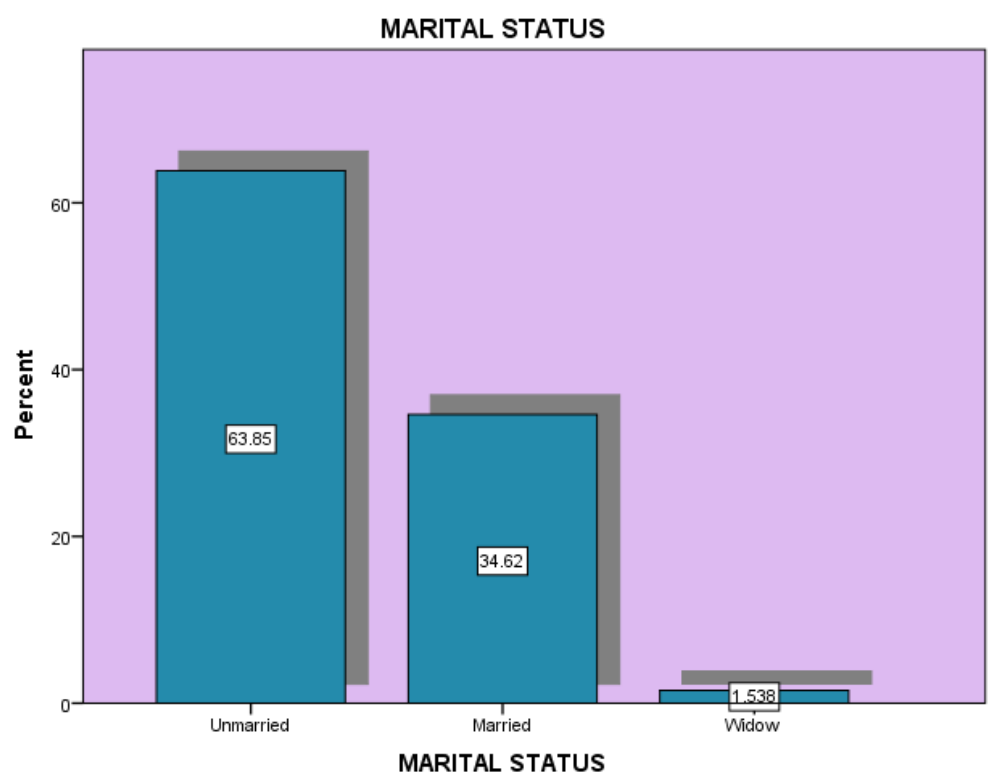

Figure: 3

4. Religion

\begin{tabular}{|l|l|l|l|l|l|}
\hline \multicolumn{2}{|c|}{ Religion } & Frequency & $\%$ & Val \% & Cum \% \\
\hline \multirow{4}{*}{ Val } & Hindu & 73 & 56.2 & 56.2 & 56.2 \\
\cline { 2 - 6 } & Muslim & 39 & 30.0 & 30.0 & 86.2 \\
\cline { 2 - 6 } & Jain & 18 & 13.8 & 13.8 & 100.0 \\
\cline { 2 - 6 } & Total & 130 & 100.0 & 100.0 & \\
\hline
\end{tabular}

Table: 4

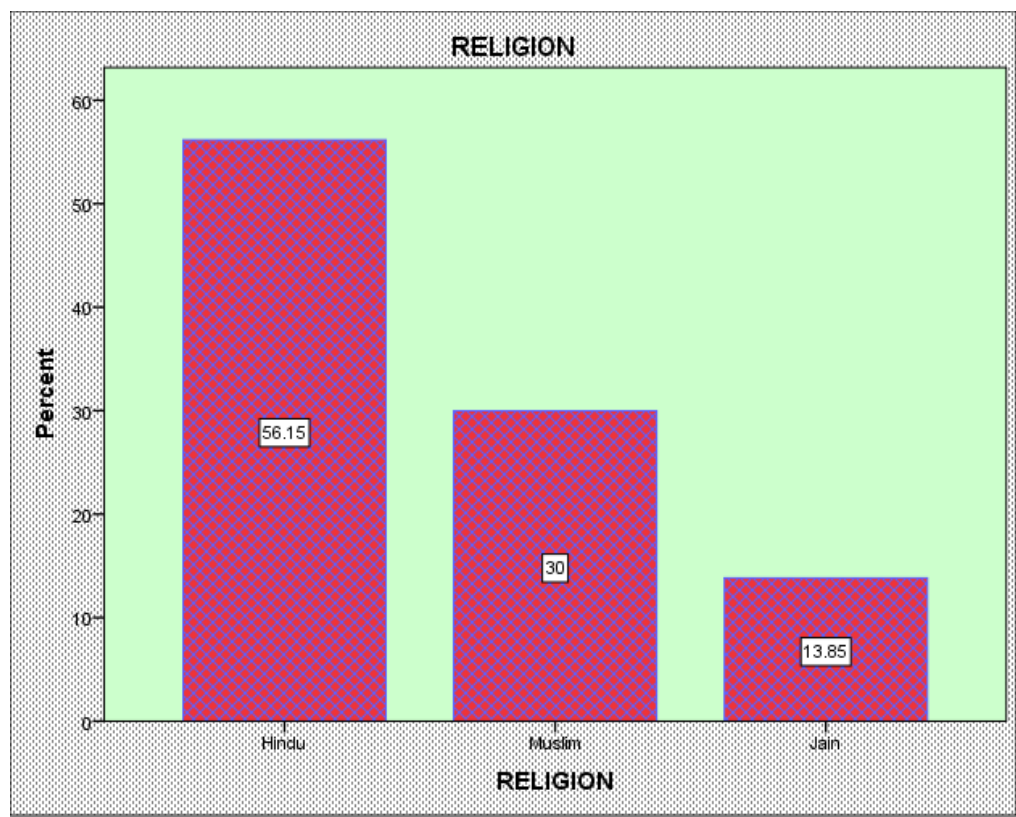

Figure: 4 
An Experimental Evaluation on the awareness of "Pradhan Mantri Jan Aryogya Yojna - Ayushmaan Bharat" (PMJAY-AB) Scheme among residents of Moradabad, Uttar Pradesh

\section{EDUCATION QUALIFICATIONS}

\begin{tabular}{|l|l|l|l|l|l|}
\hline \multicolumn{2}{|c|}{} & Frequency & $\%$ & Val \% & Cum \% \\
\hline \multirow{4}{*}{ Val } & PRE_GRAD & 21 & 16.2 & 16.2 & 16.2 \\
\cline { 2 - 6 } & GRADUATION & 65 & 50.0 & 50.0 & 66.2 \\
\cline { 2 - 6 } & POST_GRAD & 44 & 33.8 & 33.8 & 100.0 \\
\cline { 2 - 6 } & Total & 130 & 100.0 & 100.0 & \\
\hline
\end{tabular}

Table: 5

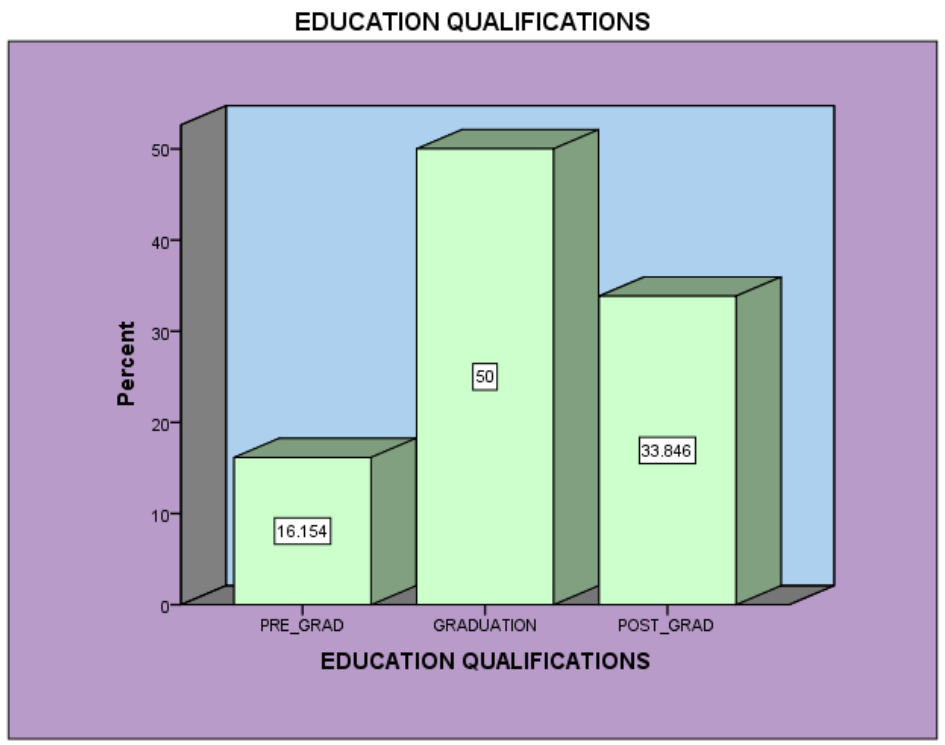

Figure: 5

\section{OCCUPATION}

\begin{tabular}{|l|l|l|l|l|l|}
\hline \multicolumn{2}{|c|}{} & Frequency & $\%$ & Val \% & Cum \% \\
\hline \multirow{4}{*}{ Val } & House_wife & 10 & 7.7 & 7.7 & 7.7 \\
\cline { 2 - 6 } & Student & 37 & 28.5 & 28.5 & 36.2 \\
\cline { 2 - 6 } & Business & 32 & 24.6 & 24.6 & 60.8 \\
\cline { 2 - 6 } & Service & 51 & 39.2 & 39.2 & 100.0 \\
\cline { 2 - 6 } & Total & 130 & 100.0 & 100.0 & \\
\hline
\end{tabular}

Table: 6

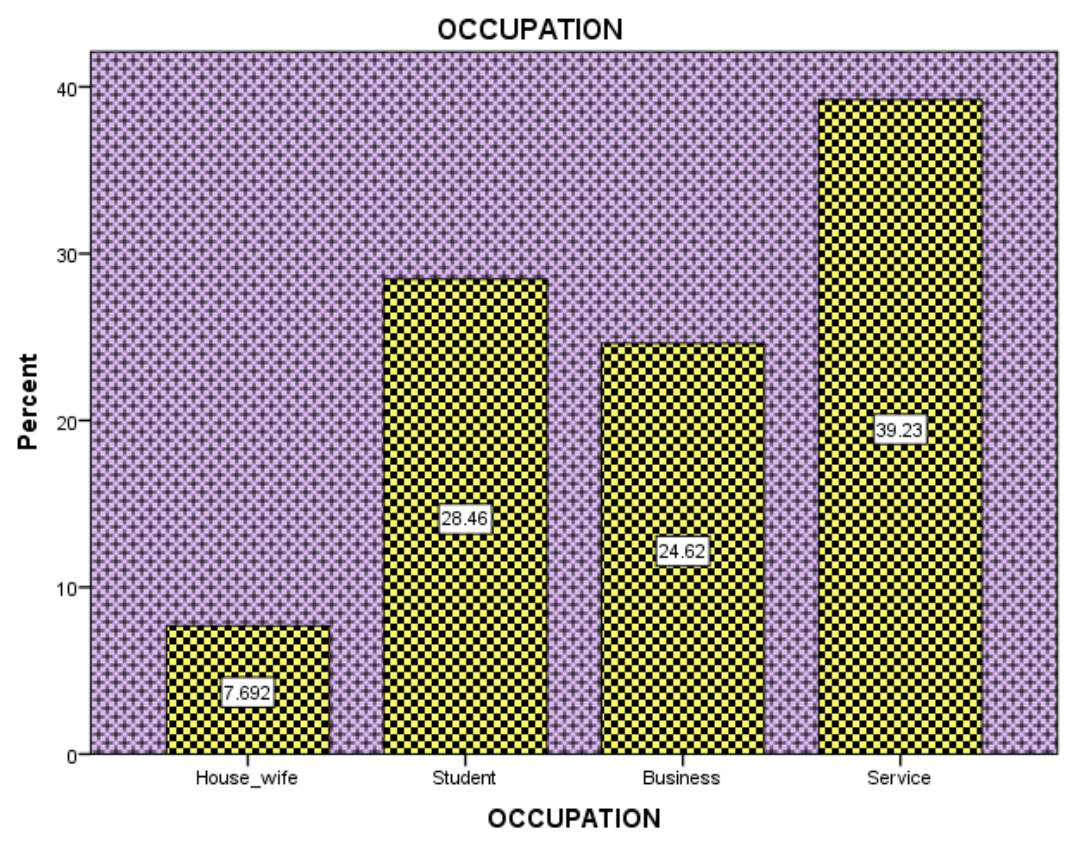

Figure: 6 
7. INCOME

\begin{tabular}{|l|l|l|l|l|l|}
\hline \multicolumn{2}{|c|}{ Income } & Frequency & $\%$ & Val \% & Cum \% \\
\hline \multirow{4}{*}{} & Very Low & 53 & 40.8 & 40.8 & 40.8 \\
\cline { 2 - 6 } & Low & 17 & 13.1 & 13.1 & 53.8 \\
\cline { 2 - 6 } & Average & 21 & 16.2 & 16.2 & 70.0 \\
\cline { 2 - 6 } & High & 22 & 16.9 & 16.9 & 86.9 \\
\cline { 2 - 6 } & Very High & 17 & 13.1 & 13.1 & 100.0 \\
\cline { 2 - 6 } & Total & 130 & 100.0 & 100.0 & \\
\hline
\end{tabular}

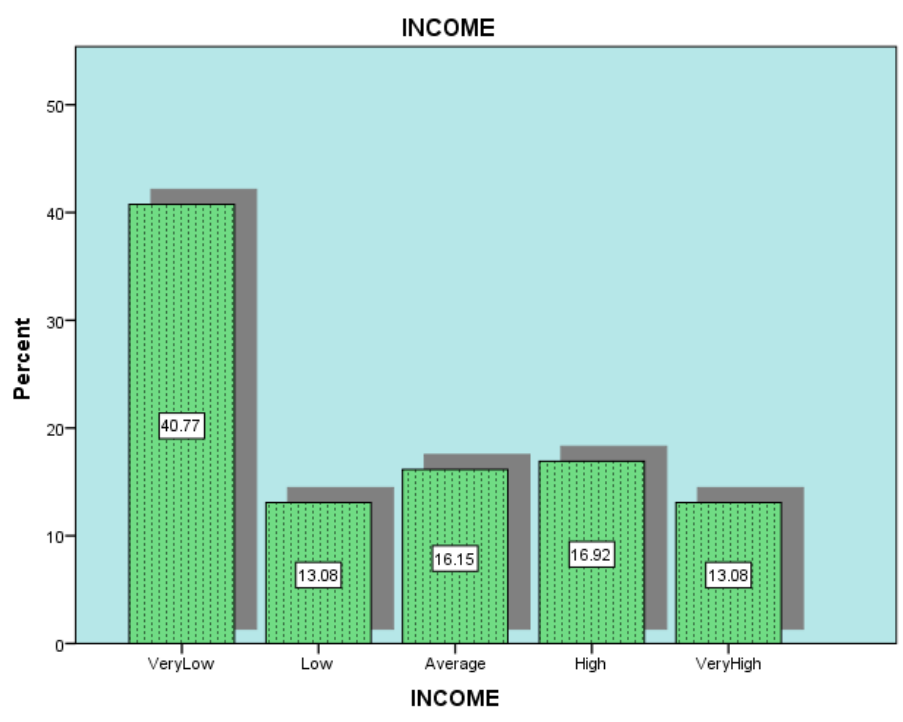

Figure: 7

8. PMJAY - AB Awareness Category

\begin{tabular}{|l|l|l|l|l|l|}
\hline \multicolumn{2}{|c|}{} & Frq & $\%$ & Val \% & Cum \% \\
\hline \multirow{5}{*}{ Val } & AVERAGE & 35 & 26.9 & 26.9 & 26.9 \\
\cline { 2 - 6 } & HIGH & 34 & 26.2 & 26.2 & 53.1 \\
\cline { 2 - 6 } & LOW & 19 & 14.6 & 14.6 & 67.7 \\
\cline { 2 - 6 } & VERYHIGH & 30 & 23.1 & 23.1 & 90.8 \\
\cline { 2 - 6 } & VERYLOW & 12 & 9.2 & 9.2 & 100.0 \\
\cline { 2 - 6 } & Total & 130 & 100.0 & 100.0 & \\
\hline
\end{tabular}

Table: 8

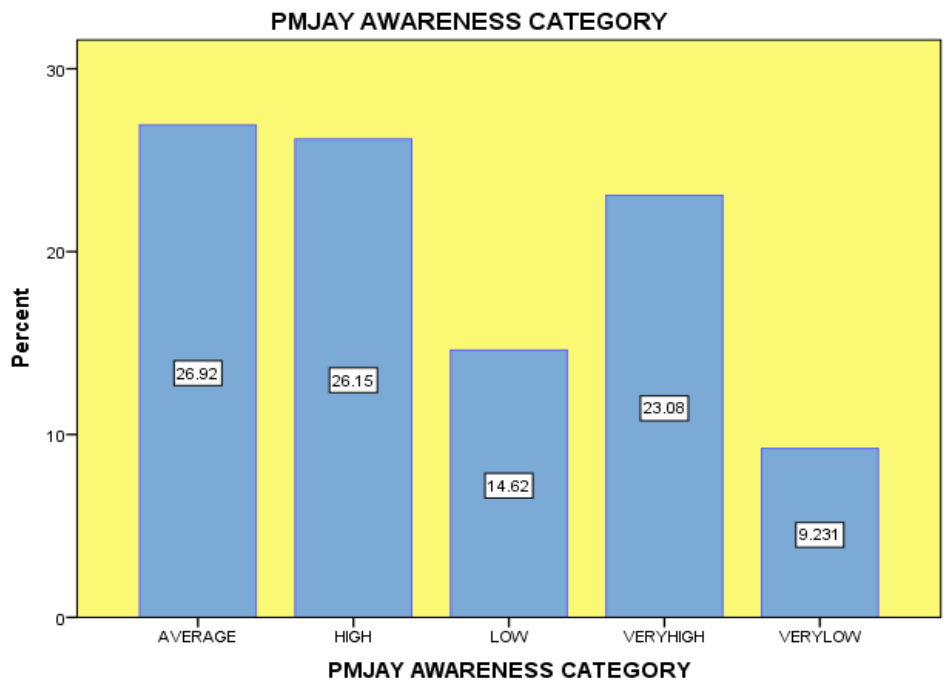

Figure: 8 
Result \& Interpretation of Descriptive Analysis:

A community based empirical study provided the following descriptive analysis:-

Out of total number of 130 respondents:

1. Gender: Male (75) and Female (55) are 57.7\% and $42.3 \%$ respectively,

2. Age Groups: Young Generation (20-30yrs) - 17 (13.1\%), Adult Generation $1^{\text {st }}(30-40 \mathrm{yrs})-56$ $(43.1 \%)$, Adult Generation $2^{\text {nd }}(40-50 \mathrm{yrs})-37$ (28.5\%), Old Baby Boomers (50-60yrs) - 16 (12.3\%), Vey Old Baby Boomers (above 60) - 4 (3.1\%),

3. Marital Status: Unmarried $83(63.8 \%)$, married $45(34.5 \%)$ and widow $2(1.5 \%)$,

4. Religion groups: Hindu 73 (56.2), Muslims 39 (30\%) and Jain 18 (13.8)

5. Education Qualification: Pre graduates 21 $(16.2 \%)$, Graduates $65(50 \%)$ and Post graduates $44(33.8 \%)$,

6. Occupation: Housewife 10 (7.7\%), Student 37 (28.5\%), Business 32 (24.6\%), and Services 51 (39.2\%),

7. Income: Very low 53 (40.8\%), Low 17 (13.1\%), Average 21 (16.2), High 22 (16.9\%) and Very High 17 (13.1\%),
8. PMJAY-AB awareness category: The awareness of Pradhan Mantra Jan Aryogya Yojna was found to be Very High 30 (23.1\%), High 34 (26.2\%), Average 35 (26.9), Low 19 $(14.6 \%)$ and Very Low $12(9.2 \%)$ of the respondents.

\section{B.Chi-SQuare Test}

1. Relationship between gender group and PMJAY-AB Awareness:

$H_{1 N}$ : There is no significant relationship between Gender Group and PMJAY-AB Awareness. $H_{1 A}$ : There is a significant relationship between Gender Group and PMJAY-AB Awareness.

To prove, a descriptive analysis is done by making Cross-tabulation and tested with Chi-square test.

\begin{tabular}{|c|c|c|c|c|c|c|c|c|}
\hline & \multicolumn{5}{|c|}{ PMJAY AWARENESS } & \multirow[t]{2}{*}{ Total } \\
\hline & & & AVERAGE & HIGH & LOW & VERYHIGH & VERYLOW & \\
\hline \multirow{6}{*}{$\frac{\sqrt[r]{W}}{\sum_{\mathbb{N}}}$} & \multirow{3}{*}{$\frac{\stackrel{0}{\tilde{J}}}{\sum^{\pi}}$} & Count & 20 & 16 & 9 & 21 & 9 & 75 \\
\hline & & $\%$ within GENDER & $26.7 \%$ & $21.3 \%$ & $12.0 \%$ & $28.0 \%$ & $12.0 \%$ & $100.0 \%$ \\
\hline & & Std. Residual & .0 & -.8 & -.6 & .9 & .8 & \\
\hline & \multirow{3}{*}{ 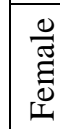 } & Count & 15 & 18 & 10 & 9 & 3 & 55 \\
\hline & & $\%$ within GENDER & $27.3 \%$ & $32.7 \%$ & $18.2 \%$ & $16.4 \%$ & $5.5 \%$ & $100.0 \%$ \\
\hline & & Std. Residual & .0 & 1.0 & .7 & -1.0 & -.9 & \\
\hline
\end{tabular}

\section{Chi-Sq-Test}

\begin{tabular}{|c|c|c|c|c|c|c|c|c|}
\hline \multicolumn{5}{|c|}{ Chi-Sq-1est } & & & & \\
\hline & & \begin{tabular}{|l|} 
Value \\
\end{tabular} & Degree of & Asymp. & & & & \\
\hline \multicolumn{2}{|c|}{ Pearson Chi-Sq } & $5.744^{\mathrm{a}}$ & 4 & .219 & & & & \\
\hline \multirow{2}{*}{ Total } & Coun & & 35 & 34 & 19 & 30 & 12 & 130 \\
\hline & $\%$ wi & ENDER & $26.9 \%$ & $26.2 \%$ & $14.6 \%$ & $23.1 \%$ & $9.2 \%$ & $100.0 \%$ \\
\hline
\end{tabular}

\section{Result \& Interpretation:}

Significant relationship between Gender Group and PMJAY-AB Awareness is not observed. As results are not significant, Null hypothesis is accepted.

$\mathrm{X}^{2}(1, \mathrm{~N}=130)=21.66, \mathrm{p}=.219$

\section{Relationship between age group and PMJAY-AB Awareness.}

$\mathrm{H}_{2 \mathrm{~N}}$ : There is no significant relationship between Age Group and PMJAY-AB Awareness.

$\mathrm{H}_{2 \mathrm{~A}}$ : There is a significant relationship between Age Group and PMJAY-AB Awareness.

To prove, a descriptive analysis is done by making Cross tabulation and tested with

Chi-square test. 


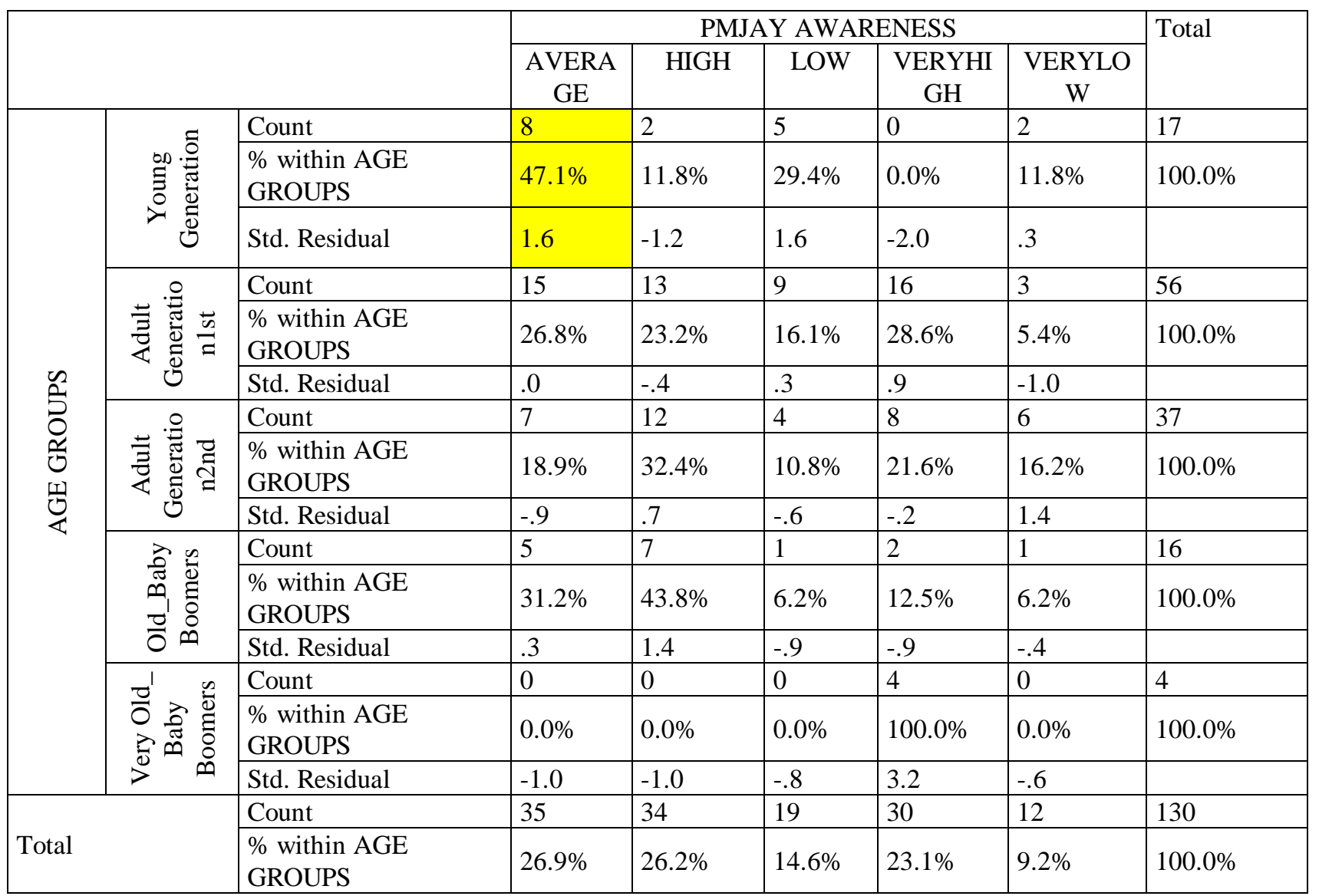

\begin{tabular}{|l|l|l|l|}
\hline \multicolumn{5}{|l|}{ Chi-Sq-Test } & Value & $\begin{array}{l}\text { Degree of } \\
\text { Freedom }\end{array}$ & $\begin{array}{l}\text { Asymp. Sig. } \\
\text { (2-sided) }\end{array}$ \\
\hline Pearson Chi-Sq & $33.232^{\mathrm{a}}$ & 16 & .007 \\
\hline
\end{tabular}

\section{Result \& Interpretation:}

There are significant evidence to prove relationship between Age Group and PMJAY-AB Awareness. As results are significant, Null hypothesis is rejected. $\mathrm{X}^{2}(1, \mathrm{~N}=130)$ $=33.23, \mathrm{p}=.007$ Young_GenX Group is significantly related to Average PMJAY Awareness with $47.1 \%$ Within_Grp and Std. Residual 1.6 most near to 1.96

\section{Relationship between marital status and PMJAY-AB Awareness}

$\mathrm{H}_{3 \mathrm{~N}}$ : There is no significant relationship between Marital Status and PMJAY -AB Awareness.

$\mathbf{H}_{3 \mathrm{~A}}$ : There is a significant relationship between Marital Status and PMJAY-AB Awareness.

To prove, a descriptive analysis is done by making Cross tabulation and tested with Chi-square test.

\begin{tabular}{|c|c|c|c|c|c|c|c|c|}
\hline & \multicolumn{5}{|c|}{ PMJAY AWARENESS } & \multirow[t]{2}{*}{ Total } \\
\hline & & & AVERAGE & HIGH & LOW & VERYHIGH & VERYLOW & \\
\hline \multirow{9}{*}{ 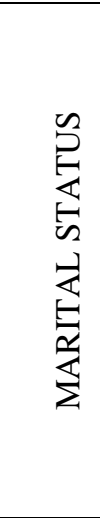 } & \multirow{3}{*}{ 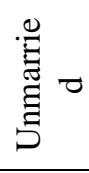 } & Count & 26 & 21 & 15 & 16 & 5 & 83 \\
\hline & & Exp. Count & 22.3 & 21.7 & 12.1 & 19.2 & 7.7 & 83.0 \\
\hline & & $\begin{array}{l}\text { \% within MARITAL } \\
\text { STATUS }\end{array}$ & $31.3 \%$ & $25.3 \%$ & $18.1 \%$ & $19.3 \%$ & $6.0 \%$ & $100.0 \%$ \\
\hline & \multirow{3}{*}{ 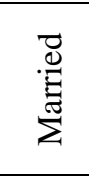 } & Count & 9 & 13 & 4 & 12 & 7 & 45 \\
\hline & & Exp. Count & 12.1 & 11.8 & 6.6 & 10.4 & 4.2 & 45.0 \\
\hline & & $\begin{array}{l}\% \text { within MARITAL } \\
\text { STATUS }\end{array}$ & $20.0 \%$ & $28.9 \%$ & $8.9 \%$ & $26.7 \%$ & $15.6 \%$ & $100.0 \%$ \\
\hline & \multirow{3}{*}{$\begin{array}{l}3 \\
3 \\
3 \\
3\end{array}$} & Count & 0 & 0 & 0 & 2 & 0 & 2 \\
\hline & & Exp. Count & .5 & .5 & .3 & .5 & .2 & 2.0 \\
\hline & & $\begin{array}{l}\% \text { within MARITAL } \\
\text { STATUS }\end{array}$ & $0.0 \%$ & $0.0 \%$ & $0.0 \%$ & $100.0 \%$ & $0.0 \%$ & $100.0 \%$ \\
\hline \multirow{3}{*}{\multicolumn{2}{|c|}{ Total }} & Count & 35 & 34 & 19 & 30 & 12 & 130 \\
\hline & & Exp. Count & 35.0 & 34.0 & 19.0 & 30.0 & 12.0 & 130.0 \\
\hline & & $\begin{array}{l}\% \text { within MARITAL } \\
\text { STATUS }\end{array}$ & $26.9 \%$ & $26.2 \%$ & $14.6 \%$ & $23.1 \%$ & $9.2 \%$ & $100.0 \%$ \\
\hline
\end{tabular}


An Experimental Evaluation on the awareness of "Pradhan Mantri Jan Aryogya Yojna - Ayushmaan Bharat" (PMJAY-AB) Scheme among residents of Moradabad, Uttar Pradesh

\begin{tabular}{|c|c|c|c|}
\hline \multicolumn{4}{|c|}{ Chi-Sq-Test } \\
\hline & Value & $\begin{array}{l}\text { Degree_ } \\
\text { Freedom } \\
\end{array}$ & $\begin{array}{l}\text { Asymp. Sig. } \\
\text { (2-sided) }\end{array}$ \\
\hline $\begin{array}{l}\text { Pearson } \\
\text { Chi-Sq }\end{array}$ & $13.551^{\mathrm{a}}$ & 8 & .094 \\
\hline
\end{tabular}

Result \& Interpretation:

Significant relationship between Marital Status

and PMJAY-AB Awareness is not observed. As results are not significant, Null hypothesis is accepted.

$\mathrm{X}^{2}(1, \mathrm{~N}=130)=13.55, \mathrm{p}=.094$

\section{Relationship between religion and PMJAY-AB Awareness}

$\mathrm{H}_{4 \mathrm{~N}}$ : There is no significant relationship between Religion and PMJAY-AB Awareness.

$\mathrm{H}_{4 \mathrm{~A}}$ : There is a significant relationship between Religion and PMJAY-AB Awareness.

To prove, a descriptive analysis is done by making Cross tabulation and tested with Chi-square test.

\begin{tabular}{|c|c|c|c|c|c|c|c|c|}
\hline & \multicolumn{5}{|c|}{ PMJAY AWARENESS } & \multirow{2}{*}{ Total } \\
\hline & & & AVERAGE & $\mathrm{HIGH}$ & LOW & VERYHIGH & VERYLOW & \\
\hline \multirow{9}{*}{ 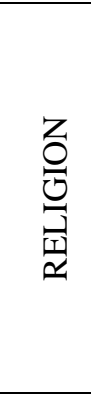 } & \multirow{3}{*}{$\underset{\Xi}{\Xi}$} & Count & 16 & 19 & 6 & 23 & 9 & 73 \\
\hline & & Exp. Count & 19.7 & 19.1 & 10.7 & 16.8 & 6.7 & 73.0 \\
\hline & & $\%$ within RELIGION & $21.9 \%$ & $26.0 \%$ & $8.2 \%$ & $31.5 \%$ & $12.3 \%$ & $100.0 \%$ \\
\hline & \multirow{3}{*}{$\sum_{\bar{\Sigma}}^{\bar{\Xi}} \Xi$} & Count & 15 & 9 & 9 & 4 & 2 & 39 \\
\hline & & Exp. Count & 10.5 & 10.2 & 5.7 & 9.0 & 3.6 & 39.0 \\
\hline & & $\%$ within RELIGION & $38.5 \%$ & $23.1 \%$ & $23.1 \%$ & $10.3 \%$ & $5.1 \%$ & $100.0 \%$ \\
\hline & \multirow{3}{*}{. } & Count & 4 & 6 & 4 & 3 & 1 & 18 \\
\hline & & Exp. Count & 4.8 & 4.7 & 2.6 & 4.2 & 1.7 & 18.0 \\
\hline & & $\%$ within RELIGION & $22.2 \%$ & $33.3 \%$ & $22.2 \%$ & $16.7 \%$ & $5.6 \%$ & $100.0 \%$ \\
\hline \multirow{3}{*}{\multicolumn{2}{|c|}{ Total }} & Count & 35 & 34 & 19 & 30 & 12 & 130 \\
\hline & & Exp. Count & 35.0 & 34.0 & 19.0 & 30.0 & 12.0 & 130.0 \\
\hline & & $\%$ within RELIGION & $26.9 \%$ & $26.2 \%$ & $14.6 \%$ & $23.1 \%$ & $9.2 \%$ & $100.0 \%$ \\
\hline
\end{tabular}

\begin{tabular}{|c|c|c|c|}
\hline \multicolumn{4}{|c|}{ Chi-Sq-Test } \\
\hline & Value & $\begin{array}{l}\text { Degree } \\
\text { Freedom }\end{array}$ & $\begin{array}{l}\text { Asymp. Sig. } \\
\text { (2-sided) }\end{array}$ \\
\hline $\begin{array}{l}\text { Pearson } \\
\text { Chi-Sq }\end{array}$ & $14.998^{\mathrm{a}}$ & 8 & .059 \\
\hline
\end{tabular}

\section{Result \& Interpretation:}

Significant relationship between Religion and PMJAY - AB Awareness is not observed. As results are not significant, Null hypothesis is accepted. $\mathrm{X}^{2}(1, \mathrm{~N}=130)=14.99, \mathrm{p}=.059$

5. Relationship between education qualification and PMJAY-AB Awareness:

$\mathrm{H}_{5 \mathrm{~N}}$ : There is no significant relationship between Education Qualification and PMJAY-AB Awareness

$\mathrm{H}_{5 \mathrm{~A}}$ : There is a significant relationship between Education Qualification and PMJAY-AB Awareness

To prove, a descriptive analysis is done by making Cross tabulation and tested with Chi-square test.

\begin{tabular}{|c|c|c|c|c|c|c|c|c|}
\hline & \multicolumn{5}{|c|}{ PMJAY AWARENESS } & \multirow[t]{2}{*}{ Total } \\
\hline & & & AVERAGE & HIGH & LOW & VERYHIGH & VERYLOW & \\
\hline \multirow{9}{*}{ 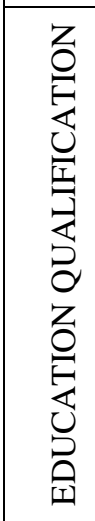 } & \multirow{3}{*}{ 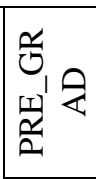 } & Count & 8 & 5 & 4 & 1 & 3 & 21 \\
\hline & & Exp. Count & 5.7 & 5.5 & 3.1 & 4.8 & 1.9 & 21.0 \\
\hline & & $\begin{array}{l}\% \text { within EDUCATION } \\
\text { QUALIFICATIONS } \\
\end{array}$ & $38.1 \%$ & $23.8 \%$ & $19.0 \%$ & $4.8 \%$ & $14.3 \%$ & $100.0 \%$ \\
\hline & \multirow{3}{*}{ 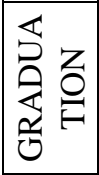 } & Count & 15 & 13 & 9 & 22 & 6 & 65 \\
\hline & & Exp. Count & 17.5 & 17.0 & 9.5 & 15.0 & 6.0 & 65.0 \\
\hline & & $\begin{array}{l}\% \quad \text { within } \quad \text { EDUCATION } \\
\text { QUALIFICATIONS }\end{array}$ & $23.1 \%$ & $20.0 \%$ & $13.8 \%$ & $33.8 \%$ & $9.2 \%$ & $100.0 \%$ \\
\hline & \multirow{3}{*}{ 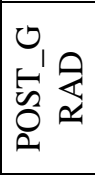 } & Count & 12 & 16 & 6 & 7 & 3 & 44 \\
\hline & & Exp. Count & 11.8 & 11.5 & 6.4 & 10.2 & 4.1 & 44.0 \\
\hline & & $\begin{array}{l}\% \quad \text { within } \quad \text { EDUCATION } \\
\text { QUALIFICATIONS }\end{array}$ & $27.3 \%$ & $36.4 \%$ & $13.6 \%$ & $15.9 \%$ & $6.8 \%$ & $100.0 \%$ \\
\hline \multirow{3}{*}{ Total } & & Count & 35 & 34 & 19 & 30 & 12 & 130 \\
\hline & & Exp. Count & 35.0 & 34.0 & 19.0 & 30.0 & 12.0 & 130.0 \\
\hline & & $\begin{array}{l}\% \quad \text { within } \quad \text { EDUCATION } \\
\text { QUALIFICATIONS }\end{array}$ & $26.9 \%$ & $26.2 \%$ & $14.6 \%$ & $23.1 \%$ & $9.2 \%$ & $100.0 \%$ \\
\hline
\end{tabular}




\begin{tabular}{|c|c|c|c|}
\hline \multicolumn{4}{|l|}{ Chi-Sq-Test } \\
\hline & Value & $\begin{array}{l}\text { Degree of } \\
\text { Freedom }\end{array}$ & $\begin{array}{l}\text { Asymp. Sig. } \\
\text { (2-sided) }\end{array}$ \\
\hline Pearson Chi-Sq & $12.567^{\mathrm{a}}$ & 8 & .128 \\
\hline
\end{tabular}

Result \& Interpretation:

Significant relationship between Education Qualification and PMJAY-AB Awareness is not observed. As results are not significant, Null hypothesis is accepted. $\mathrm{X}^{2}(1, \mathrm{~N}=130)=12.57, \mathrm{p}=.128$

\section{Relationship between occupation and PMJAY-AB Awareness:}

$\mathrm{H}_{6 \mathrm{~N}}$ : There is no significant relationship between Occupation and PMJAY-AB Awareness.

$\mathrm{H}_{6 \mathrm{~A}}$ : There is a significant relationship between Occupation and PMJAY-AB Awareness.

To prove, a descriptive analysis is done by making Cross tabulation and tested with Chi-square test.

\begin{tabular}{|c|c|c|c|c|c|c|c|c|}
\hline & \multicolumn{5}{|c|}{ PMJAY AWARENESS } & \multirow[t]{2}{*}{ Total } \\
\hline & & & $\begin{array}{c}\text { AVERAG } \\
\text { E }\end{array}$ & $\mathrm{HIGH}$ & LOW & VERYHIGH & VERYLOW & \\
\hline \multirow{12}{*}{ 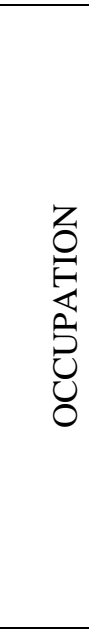 } & \multirow{3}{*}{$\begin{array}{l}\mathscr{0} \\
\stackrel{0}{3} \\
0 \\
0 \\
0 \\
0 \\
1\end{array}$} & Count & 5 & 4 & 1 & 0 & 0 & 10 \\
\hline & & Exp. Count & 2.7 & 2.6 & 1.5 & 2.3 & .9 & 10.0 \\
\hline & & $\%$ within OCCUPATION & $50.0 \%$ & $40.0 \%$ & $10.0 \%$ & $0.0 \%$ & $0.0 \%$ & $100.0 \%$ \\
\hline & \multirow{3}{*}{$\stackrel{\bar{d}}{\tilde{D}}_{\tilde{n}}$} & Count & 7 & 6 & 8 & 11 & 5 & 37 \\
\hline & & Exp. Count & 10.0 & 9.7 & 5.4 & 8.5 & 3.4 & 37.0 \\
\hline & & $\%$ within OCCUPATION & $18.9 \%$ & $16.2 \%$ & $21.6 \%$ & $29.7 \%$ & $13.5 \%$ & $100.0 \%$ \\
\hline & \multirow{3}{*}{$\begin{array}{l}\mathscr{n} \\
\stackrel{0}{\Xi} \\
\stackrel{0}{=}\end{array}$} & Count & 8 & 8 & 3 & 11 & 2 & 32 \\
\hline & & Exp. Count & 8.6 & 8.4 & 4.7 & 7.4 & 3.0 & 32.0 \\
\hline & & $\%$ within OCCUPATION & $25.0 \%$ & $25.0 \%$ & $9.4 \%$ & $34.4 \%$ & $6.2 \%$ & $100.0 \%$ \\
\hline & \multirow{3}{*}{$\sum_{0}^{0}$} & Count & 15 & 16 & 7 & 8 & 5 & 51 \\
\hline & & Exp. Count & 13.7 & 13.3 & 7.5 & 11.8 & 4.7 & 51.0 \\
\hline & & $\%$ within OCCUPATION & $29.4 \%$ & $31.4 \%$ & $13.7 \%$ & $15.7 \%$ & $9.8 \%$ & $100.0 \%$ \\
\hline \multirow{3}{*}{\multicolumn{2}{|c|}{ Total }} & Count & 35 & 34 & 19 & 30 & 12 & 130 \\
\hline & & Exp. Count & 35.0 & 34.0 & 19.0 & 30.0 & 12.0 & 130.0 \\
\hline & & $\%$ within OCCUPATION & $26.9 \%$ & $26.2 \%$ & $14.6 \%$ & $23.1 \%$ & $9.2 \%$ & $100.0 \%$ \\
\hline
\end{tabular}

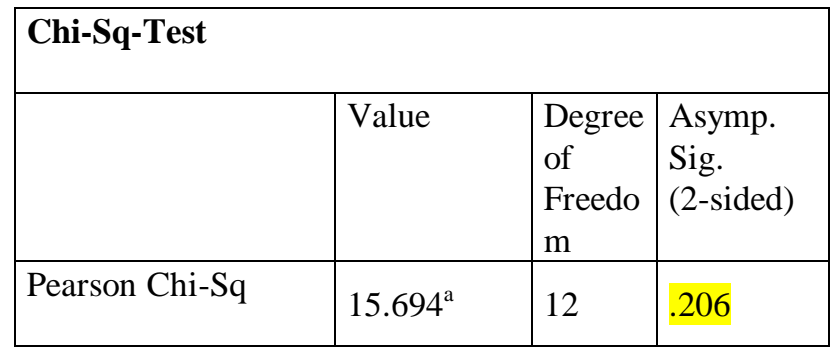

Result \& Interpretation:

Significant relationship between Occupation and PMJAY-AB Awareness is not observed.

As results are not significant, Null hypothesis is accepted. $\mathrm{X}^{2}$ $(1, \mathrm{~N}=130)=15.69, \mathrm{p}=.206$

7. Relationship between income and PMJAY-AB Awareness:

$\mathrm{H}_{7 \mathrm{~N}}$ : There is no significant relationship between Income and PMJAY-AB Awareness.

$\mathrm{H}_{7 \mathrm{~A}}$ : There is a significant relationship between Income and PMJAY-AB Awareness.

To prove a descriptive analysis is done by making Cross tabulation and tested with Chi-square test.

\begin{tabular}{|c|c|c|c|c|c|c|c|c|}
\hline & \multicolumn{5}{|c|}{ PMJAY AWARENESS } & \multirow[t]{2}{*}{ Total } \\
\hline & & & AVERAGE & HIGH & LOW & VERYHIGH & VERYLOW & \\
\hline \multirow{8}{*}{$\begin{array}{l}\sum_{0}^{ \pm} \\
Z \\
Z\end{array}$} & \multirow{3}{*}{$\stackrel{\overrightarrow{0}}{3}$} & Count & 12 & 12 & 11 & 12 & 6 & 53 \\
\hline & & Exp. Count & 14.3 & 13.9 & 7.7 & 12.2 & 4.9 & 53.0 \\
\hline & & $\%$ within INCOME & $22.6 \%$ & $22.6 \%$ & $20.8 \%$ & $22.6 \%$ & $11.3 \%$ & $100.0 \%$ \\
\hline & \multirow{3}{*}{ 高 } & Count & 5 & 4 & 1 & 6 & 1 & 17 \\
\hline & & Exp. Count & 4.6 & 4.4 & 2.5 & 3.9 & 1.6 & 17.0 \\
\hline & & $\%$ within INCOME & $29.4 \%$ & $23.5 \%$ & $5.9 \%$ & $35.3 \%$ & $5.9 \%$ & $100.0 \%$ \\
\hline & \multirow{2}{*}{ 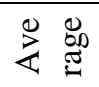 } & Count & 8 & 6 & 1 & 6 & 0 & 21 \\
\hline & & Exp. Count & 5.7 & 5.5 & 3.1 & 4.8 & 1.9 & 21.0 \\
\hline
\end{tabular}


An Experimental Evaluation on the awareness of "Pradhan Mantri Jan Aryogya Yojna - Ayushmaan Bharat" (PMJAY-AB) Scheme among residents of Moradabad, Uttar Pradesh

\begin{tabular}{|c|c|c|c|c|c|c|c|c|}
\hline & & $\%$ within INCOME & $38.1 \%$ & $28.6 \%$ & $4.8 \%$ & $28.6 \%$ & $0.0 \%$ & $100.0 \%$ \\
\hline & & Count & 7 & 3 & 6 & 4 & 2 & 22 \\
\hline & $=0$ & Exp. Count & 5.9 & 5.8 & 3.2 & 5.1 & 2.0 & 22.0 \\
\hline & & $\%$ within INCOME & $31.8 \%$ & $13.6 \%$ & $27.3 \%$ & $18.2 \%$ & $9.1 \%$ & $100.0 \%$ \\
\hline & & Count & 3 & 9 & 0 & 2 & 3 & 17 \\
\hline & 00 & Exp. Count & 4.6 & 4.4 & 2.5 & 3.9 & 1.6 & 17.0 \\
\hline & & $\%$ within INCOME & $17.6 \%$ & $52.9 \%$ & $0.0 \%$ & $11.8 \%$ & $17.6 \%$ & $100.0 \%$ \\
\hline & & Count & 35 & 34 & 19 & 30 & 12 & 130 \\
\hline Total & & Exp. Count & 35.0 & 34.0 & 19.0 & 30.0 & 12.0 & 130.0 \\
\hline & & $\%$ within INCOME & $26.9 \%$ & $26.2 \%$ & $14.6 \%$ & $23.1 \%$ & $9.2 \%$ & $100.0 \%$ \\
\hline
\end{tabular}

\begin{tabular}{|c|c|c|c|}
\hline \multicolumn{4}{|l|}{ Chi-Sq-Test } \\
\hline & Value & $\begin{array}{l}\text { Degree of } \\
\text { Freedom }\end{array}$ & $\begin{array}{l}\text { Asymp. Sig. } \\
\text { (2-sided) }\end{array}$ \\
\hline Pearson Chi-Sq & $23.232^{\mathrm{a}}$ & 16 & .108 \\
\hline
\end{tabular}

\section{Result \& Interpretation:}

Significant relationship between Income and PMJAY Awareness Category is not observed.

As results are not significant, Null hypothesis is accepted. $\mathrm{X}^{2}(1, \mathrm{~N}=130)=23.23, \mathrm{p}=.108$

\section{C.ANALYSIS OF VARIANCE (ANOVA) \& POST HOC TEST}

8. An effect of Gender on PMJAY-AB Awareness: Cannot be applied.

9. An effect of Age on PMJAY - AB Awareness.

$\mathrm{H}_{9 \mathrm{~N}}$ : Age does not have an effect on PMJAY - AB Awareness.

$\mathrm{H}_{9 \mathrm{~A}}$ : Age have an effect on PMJAY- AB Awareness Score.

To prove that PMJAY- AB Awareness is significantly influenced by different Age groups, test of Analysis of Variance (ANOVA) is conducted.

\begin{tabular}{|c|c|c|c|c|c|c|c|c|}
\hline \multicolumn{9}{|c|}{ PMJAY-AB AWARENESS } \\
\hline & \multirow[t]{2}{*}{$\mathrm{N}$} & \multirow[t]{2}{*}{ Mean } & \multirow{2}{*}{\begin{tabular}{|l} 
Std. \\
Deviation
\end{tabular}} & \multirow[t]{2}{*}{ Std. Error } & \multicolumn{2}{|c|}{$\begin{array}{l}\text { 95\% Confidence Interval for } \\
\text { Mean }\end{array}$} & \multirow[t]{2}{*}{ Min. } & \multirow[t]{2}{*}{ Max. } \\
\hline & & & & & Lower Bound & Upper Bound & & \\
\hline $\begin{array}{l}\text { Young_Generatio } \\
\text { n }\end{array}$ & 17 & 7.7059 & 2.64019 & .64034 & 6.3484 & 9.0633 & 2.00 & 13.00 \\
\hline $\begin{array}{l}\text { Adult_Generation } \\
\text { 1st }\end{array}$ & 56 & 11.1964 & 4.06502 & .54321 & 10.1078 & 12.2850 & 4.00 & 19.00 \\
\hline $\begin{array}{l}\text { Adult_Generation } \\
\text { 2nd }\end{array}$ & 37 & 10.2162 & 4.88271 & .80271 & 8.5882 & 11.8442 & 1.00 & 19.00 \\
\hline $\begin{array}{l}\text { Old_BabyBoomer } \\
\text { s }\end{array}$ & 16 & 10.8125 & 3.27045 & .81761 & 9.0698 & 12.5552 & 3.00 & 15.00 \\
\hline $\begin{array}{l}\text { VeryOld_BabyBo } \\
\text { omers }\end{array}$ & 4 & 17.2500 & .95743 & .47871 & 15.7265 & 18.7735 & 16.00 & 18.00 \\
\hline Total & 130 & 10.6000 & \begin{tabular}{|l|l}
4.30179 \\
\end{tabular} & \begin{tabular}{|l}
.37729 \\
\end{tabular} & 9.8535 & 11.3465 & 1.00 & 19.00 \\
\hline
\end{tabular}

\begin{tabular}{|l|l|l|l|l|l|}
\hline \multicolumn{6}{|l|}{ PMJAY AWARENESS * AGE GROUPS } \\
\hline & Sum of Squares & $\begin{array}{l}\text { Degree of } \\
\text { Freedom }\end{array}$ & Mean Square & F & Sig. \\
\hline Between Group & 345.374 & 4 & 86.343 & 5.286 & .001 \\
\hline Within Group & 2041.826 & 125 & 16.335 & & \\
\hline Total & 2387.200 & 129 & & & \\
\hline
\end{tabular}

PMJAY Awareness has a (significant) effect w.r.t Age groups on at the $p<.05 \_l$ level. $[F(4,125)=5.286, p=0.001]$ and Null hypothesis is rejected.Also to prove which Age group has significantly influenced Awareness of PMJAY, Post Hoc Scheffe Test is applied.

\section{Dependent Variable: PMJAY AWARENESS \\ Scheffe}

\begin{tabular}{|c|c|c|c|c|c|c|}
\hline \multirow{2}{*}{$\begin{array}{l}\text { (I) AGE } \\
\text { GROUPS }\end{array}$} & \multirow{2}{*}{ (J) AGE GROUPS } & \multirow{2}{*}{$\begin{array}{ll}\begin{array}{l}\text { Mean } \\
(\mathrm{I}-\mathrm{J})\end{array} & \text { Difference } \\
\end{array}$} & \multirow{2}{*}{ Std. Error } & \multirow[t]{2}{*}{ Sig. } & \multicolumn{2}{|c|}{ 95\% Confidence Interval } \\
\hline & & & & & Lower Bound & Upper Bound \\
\hline 100 & & -3.49055 & 1.11917 & .051 & -6.9899 & .0089 \\
\hline
\end{tabular}


International Journal of Innovative Technology and Exploring Engineering (IJITEE) ISSN: 2278-3075, Volume-8, Issue-11S2, September 2019

\begin{tabular}{|c|c|c|c|c|c|c|}
\hline & Adult_Generation2nd & -2.51033 & 1.18420 & .349 & -6.2131 & 1.1924 \\
\hline & Old_BabyBoomers & -3.10662 & 1.40775 & .307 & -7.5083 & 1.2951 \\
\hline & VeryOld_BabyBoomers & $-9.54412^{*}$ & 2.24600 & .002 & -16.5668 & -2.5214 \\
\hline \multirow{4}{*}{ 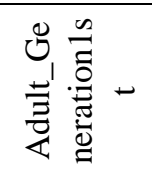 } & Young_Generation & 3.49055 & 1.11917 & .051 & -.0089 & 6.9899 \\
\hline & Adult_Generation2nd & .98021 & .85625 & .859 & -1.6971 & 3.6575 \\
\hline & Old_BabyBoomers & .38393 & 1.14569 & .998 & -3.1984 & 3.9662 \\
\hline & VeryOld_BabyBoomers & -6.05357 & 2.09173 & .086 & -12.5939 & .4868 \\
\hline \multirow{4}{*}{ 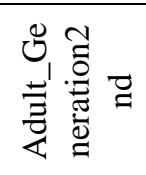 } & Young_Generation & 2.51033 & 1.18420 & .349 & -1.1924 & 6.2131 \\
\hline & Adult_Generation1st & -.98021 & .85625 & .859 & -3.6575 & 1.6971 \\
\hline & Old_BabyBoomers & -.59628 & 1.20929 & .993 & -4.3775 & 3.1849 \\
\hline & VeryOld_BabyBoomers & $-7.03378^{*}$ & 2.12724 & .032 & -13.6852 & -.3824 \\
\hline \multirow{4}{*}{ 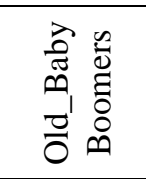 } & Young_Generation & 3.10662 & 1.40775 & .307 & -1.2951 & 7.5083 \\
\hline & Adult_Generation1st & -.38393 & 1.14569 & .998 & -3.9662 & 3.1984 \\
\hline & Adult_Generation2nd & .59628 & 1.20929 & .993 & -3.1849 & 4.3775 \\
\hline & VeryOld_BabyBoomers & -6.43750 & 2.25933 & .094 & -13.5019 & .6269 \\
\hline \multirow{4}{*}{ 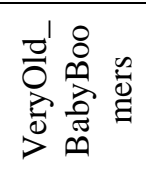 } & Young_Generation & $9.54412^{*}$ & 2.24600 & .002 & 2.5214 & 16.5668 \\
\hline & Adult_Generation 1st & 6.05357 & 2.09173 & .086 & -.4868 & 12.5939 \\
\hline & Adult_Generation2nd & $7.03378^{*}$ & 2.12724 & .032 & .3824 & 13.6852 \\
\hline & Old_BabyBoomers & 6.43750 & 2.25933 & .094 & -.6269 & 13.5019 \\
\hline
\end{tabular}

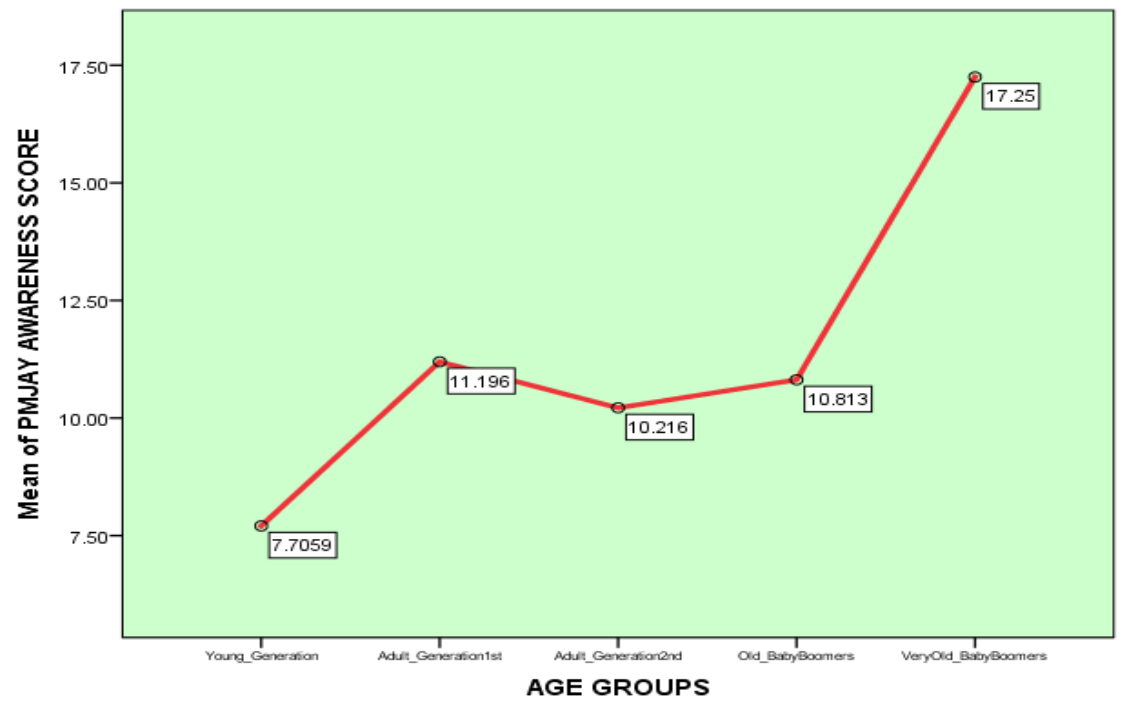

\section{Result \& Interpretation:}

The post hoc test revealed that the Scores of Awareness about PMJAY was statistically significantly different representing higher Awareness score with Age group VeryOld_BabyBoomers (17.25 \pm 0.96 marks, $\mathrm{p}=.002)$ and lower Awareness score with Age group Young_Generation $(7.71 \pm 2.64$ marks, $p=.002)$ and average Awareness score with Age group Adult_Generation2nd $(10.22 \pm 4.88$ marks, $p=.032)$. Statistically significant diff. between the Scores of Awareness regarding PMJAY among Age groups Adult_Generation1st, Adult_Generation2nd and Old_BabyBoomers (p > .05) could not be justified.

Mean plot also suggest the same results.

10. An effect of Marital status on PMJAY - AB Awareness.

$\mathrm{H}_{10 \mathrm{~N}}$ : Marital status does not has an effect on PMJAY Awareness Score.

$\mathrm{H}_{10 \mathrm{~A}}$ : Marital status has an effect on PMJAY Awareness Score.

To prove that PMJAY Awareness is significantly influenced by different Marital status, test of Analysis of Variance (ANOVA) is conducted.

\begin{tabular}{|c|c|c|c|c|c|c|c|c|}
\hline \multicolumn{9}{|c|}{ PMJAY AWARENESS } \\
\hline & \multirow[t]{2}{*}{$\mathrm{N}$} & \multirow[t]{2}{*}{ Mean } & \multirow{2}{*}{\begin{tabular}{|l|} 
Std. \\
Deviation
\end{tabular}} & \multirow[t]{2}{*}{ Std. Error } & \multicolumn{2}{|c|}{ 95\% Confidence Interval for Mean } & \multirow[t]{2}{*}{ Min. } & \multirow[t]{2}{*}{ Max. } \\
\hline & & & & & Lower Bound & Upper Bound & & \\
\hline Unmarried & 83 & 10.3855 & 3.89130 & .42713 & 9.5359 & 11.2352 & 2.00 & 19.00 \\
\hline Married & 45 & 10.6667 & 4.84299 & .72195 & 9.2117 & 12.1217 & 1.00 & 19.00 \\
\hline
\end{tabular}


An Experimental Evaluation on the awareness of "Pradhan Mantri Jan Aryogya Yojna - Ayushmaan Bharat" (PMJAY-AB) Scheme among residents of Moradabad, Uttar Pradesh

\begin{tabular}{|l|l|l|l|l|l|l|l|l|}
\hline Widow & 2 & 18.0000 & .00000 & .00000 & 18.0000 & 18.0000 & 18.00 & 18.00 \\
\hline Total & 130 & 10.6000 & 4.30179 & .37729 & 9.8535 & 11.3465 & 1.00 & 19.00 \\
\hline
\end{tabular}

\begin{tabular}{|c|c|c|c|c|c|}
\hline \multicolumn{6}{|c|}{ PMJAY AWARENESS * MARITAL STATUS } \\
\hline & $\begin{array}{l}\text { Sum of } \\
\text { Squares }\end{array}$ & $\begin{array}{l}\text { Degree of } \\
\text { Freedom }\end{array}$ & Mean Square & $\mathrm{F}$ & Sig. \\
\hline Between Group & 113.537 & 2 & 56.769 & 3.171 & .045 \\
\hline Within Group & 2273.663 & 127 & 17.903 & & \\
\hline Total & 2387.200 & 129 & & & \\
\hline
\end{tabular}

PMJAY Awareness has a (significant) effect w.r.t Marital status on at the $p<.05 \_l$ level. $[F(2,127)=3.171, p=0.045]$ and Null hypothesis is rejected.

Also to prove which Marital status group has significantly influenced Awareness of PMJAY, Post Hoc Scheffe Test is applied.

\begin{tabular}{|c|c|c|c|c|c|c|}
\hline \multicolumn{7}{|c|}{$\begin{array}{l}\text { Dependent Variable: PMJAY AWARENESS } \\
\text { Scheffe }\end{array}$} \\
\hline \multirow{2}{*}{$\begin{array}{l}\text { (I) MARITAL } \\
\text { STATUS }\end{array}$} & \multirow{2}{*}{$\begin{array}{l}\text { (J) MARITAL } \\
\text { STATUS }\end{array}$} & \multirow{2}{*}{$\begin{array}{l}\text { Mean } \text { Difference } \\
(\mathrm{I}-\mathrm{J})\end{array}$} & \multirow[t]{2}{*}{ Std. Error } & \multirow[t]{2}{*}{ Sig. } & \multicolumn{2}{|c|}{$95 \%$ Confidence Interval } \\
\hline & & & & & Lower Bound & Upper Bound \\
\hline \multirow{2}{*}{ Unmarried } & Married & -.28112 & .78329 & .938 & -2.2212 & 1.6590 \\
\hline & Widow & $-7.61446^{*}$ & 3.02773 & .046 & -15.1138 & -.1151 \\
\hline \multirow{2}{*}{ Married } & Unmarried & .28112 & .78329 & .938 & -1.6590 & 2.2212 \\
\hline & Widow & -7.33333 & 3.05766 & .060 & -14.9069 & .2402 \\
\hline \multirow{2}{*}{ Widow } & Unmarried & $7.61446^{*}$ & 3.02773 & .046 & .1151 & 15.1138 \\
\hline & Married & 7.33333 & 3.05766 & .060 & -.2402 & 14.9069 \\
\hline
\end{tabular}

*At 0.05 level, the mean differences were found statically significant

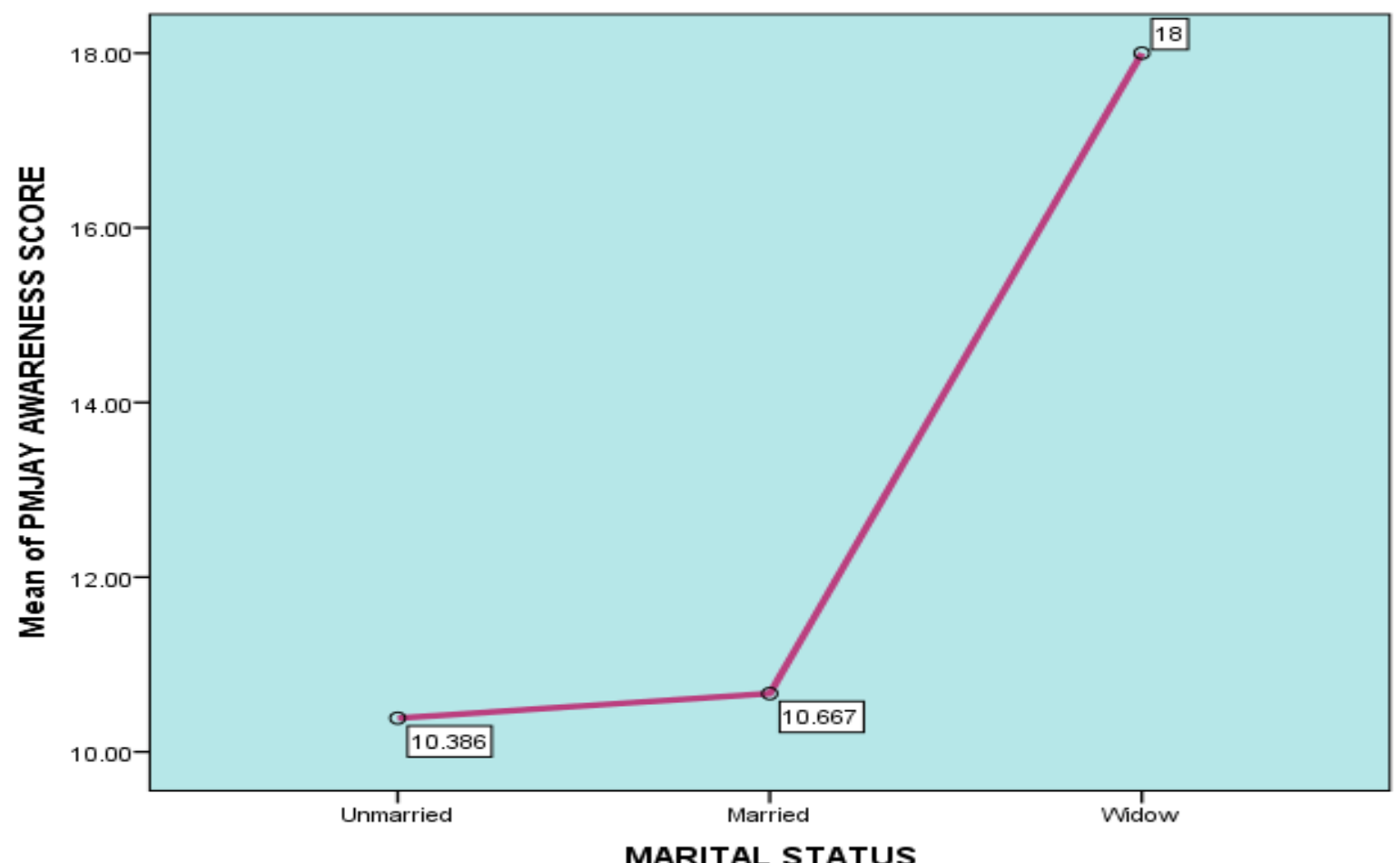

Result \& Interpretation:

The post hoc test revealed that the Scores of Awareness about PMJAY was statistically significantly different representing higher Awareness score among Widow (18 \pm 0.00 marks, $\mathrm{p}=.046)$.

Significant diff. between the Scores of Awareness regarding the Scores of Awareness regarding PMJAY among Unmarried (10.386 \pm 3.89$)$ and Married $(10.667 \pm 4.84)$ where $\mathrm{p}=.938>.05$

Mean plot also suggest the same results.

An effect of Religion on PMJAY - AB Awareness. 
$H_{11 N}$ : Religion does not has an effect on PMJAY Awareness.

$\mathrm{H}_{11 \mathrm{~A}}$ : Religion has an effect on PMJAY Awareness.

To prove that PMJAY Awareness is significantly influenced by different Religion, test of Analysis of Variance (ANOVA) is conducted.

\begin{tabular}{|c|c|c|c|c|c|}
\hline \multicolumn{6}{|c|}{ PMJAY AWARENESS SCORE * RELIGION } \\
\hline & $\begin{array}{l}\text { Sum of } \\
\text { Squares }\end{array}$ & $\begin{array}{l}\text { Degree of } \\
\text { Freedom }\end{array}$ & Mean Square & $\mathrm{F}$ & Sig. \\
\hline Between Group & 51.269 & 2 & 25.635 & 1.394 & .252 \\
\hline Within Group & 2335.931 & 127 & 18.393 & & \\
\hline Total & 2387.200 & 129 & & & \\
\hline
\end{tabular}

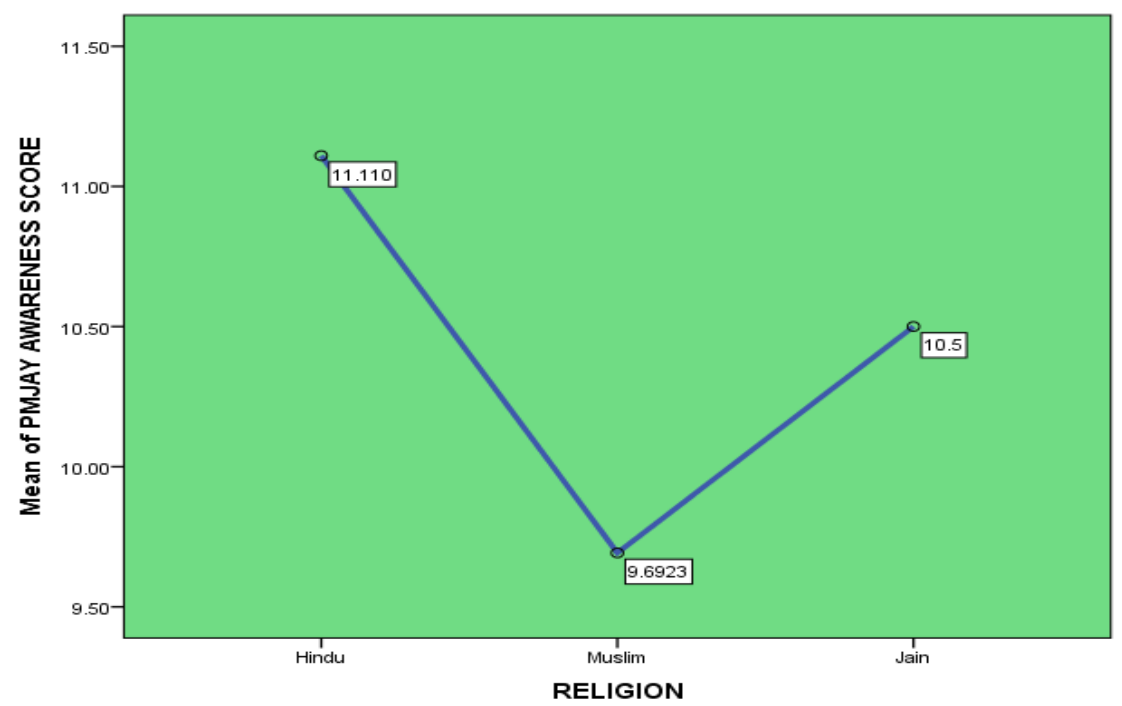

\section{Result \& Interpretation:}

There was no significant effect of Religion on PMJAY Awareness at the $p=.252>.05$

Post Hoc test cannot apply as there is no significant effect observed.

Null hypothesis is accepted. Same results are observed from mean plot too.

\section{An effect of Education Qualifications on PMJAY - AB Awareness.}

$\mathrm{H}_{12 \mathrm{~N}}$ : Education qualification does not has an effect on PMJAY Awareness.

$\mathrm{H}_{12 \mathrm{~A}}$ : Education qualification has an effect on PMJAY Awareness.

To prove that PMJAY Awareness is significantly influenced by difference in Education qualifications, test of Analysis of Variance (ANOVA) is conducted.

\begin{tabular}{|c|c|c|c|c|c|c|c|c|}
\hline \multicolumn{9}{|c|}{ PMJAY AWARENESS SCORE } \\
\hline & \multirow[t]{2}{*}{$\mathrm{N}$} & \multirow[t]{2}{*}{ Mean } & \multirow[t]{2}{*}{ Std. Deviation } & \multirow[t]{2}{*}{ Std. Error } & \multicolumn{2}{|c|}{$\begin{array}{l}\text { 95\% Confidence Interval for } \\
\text { Mean }\end{array}$} & \multirow[t]{2}{*}{ Min. } & \multirow[t]{2}{*}{ Max. } \\
\hline & & & & & Lower Bound & $\begin{array}{l}\text { Upper } \\
\text { Bound }\end{array}$ & & \\
\hline PRE_GRAD & 21 & 8.3810 & 3.73465 & .81497 & 6.6810 & 10.0809 & 1.00 & 16.00 \\
\hline GRADUATION & 65 & 11.2154 & 4.49824 & .55794 & 10.1008 & 12.3300 & 3.00 & 18.00 \\
\hline POST_GRAD & 44 & 10.7500 & 3.98908 & .60138 & 9.5372 & 11.9628 & 1.00 & 19.00 \\
\hline Total & 130 & 10.6000 & 4.30179 & .37729 & 9.8535 & 11.3465 & 1.00 & 19.00 \\
\hline
\end{tabular}

\begin{tabular}{|l|l|l|l|l|l|}
\hline PMJAY AWARENESS SCORE * EDUCATION QUALIFICATIONS \\
\hline & $\begin{array}{l}\text { Sum of } \\
\text { Squares }\end{array}$ & $\begin{array}{l}\text { Degree of } \\
\text { Freedom }\end{array}$ & Mean Square & F & Sig. \\
\hline Between Group & 129.013 & 2 & 64.507 & 3.628 & .029 \\
\hline Within Group & 2258.187 & 127 & 17.781 & & \\
\hline Total & 2387.200 & 129 & & & \\
\hline
\end{tabular}


An Experimental Evaluation on the awareness of "Pradhan Mantri Jan Aryogya Yojna - Ayushmaan Bharat" (PMJAY-AB) Scheme among residents of Moradabad, Uttar Pradesh

PMJAY Awareness has a (significant) effect w.r.t Education qualification on at the $\mathrm{p}<.05$ level. $[\mathrm{F}(2,127)=3.628$, $\mathrm{p}=0.029]$ and Null hypothesis is rejected.

Also to prove which Education qualification group has significantly influenced Awareness of PMJAY, Post Hoc Scheffe Test is applied.

\begin{tabular}{|c|c|c|c|c|c|c|}
\hline \multicolumn{7}{|c|}{$\begin{array}{l}\text { Dependent Variable: PMJAY AWARENESS SCORE } \\
\text { Scheffe }\end{array}$} \\
\hline \multirow[t]{2}{*}{$\begin{array}{l}\text { (I) EDUCATION } \\
\text { QUALIFICATIONS }\end{array}$} & \multirow[t]{2}{*}{$\begin{array}{lr}(\mathrm{J}) & \text { EDUCATION } \\
\text { QUALIFICATIONS }\end{array}$} & \multirow[t]{2}{*}{$\begin{array}{l}\text { Mean } \\
\text { Difference (I-J) }\end{array}$} & \multirow[t]{2}{*}{ Std. Error } & \multirow[t]{2}{*}{ Sig. } & \multicolumn{2}{|l|}{$\begin{array}{l}95 \% \\
\text { Interval }\end{array}$} \\
\hline & & & & & $\begin{array}{l}\text { Lower } \\
\text { Bound } \\
\end{array}$ & $\begin{array}{l}\text { Upper } \\
\text { Bound } \\
\end{array}$ \\
\hline \multirow{2}{*}{ UNDER_GRAD } & GRADUATION & $-2.83443^{*}$ & 1.05843 & .031 & -5.4561 & -.2128 \\
\hline & POST_GRAD & -2.36905 & 1.11840 & .110 & -5.1392 & .4011 \\
\hline \multirow{2}{*}{ GRADUATION } & PRE_GRAD & $2.83443^{*}$ & 1.05843 & .031 & .2128 & 5.4561 \\
\hline & POST_GRAD & .46538 & .82321 & .852 & -1.5736 & 2.5044 \\
\hline \multirow{2}{*}{ POST_GRAD } & PRE_GRAD & 2.36905 & 1.11840 & .110 & -.4011 & 5.1392 \\
\hline & GRADUATION & -.46538 & .82321 & .852 & -2.5044 & 1.5736 \\
\hline
\end{tabular}

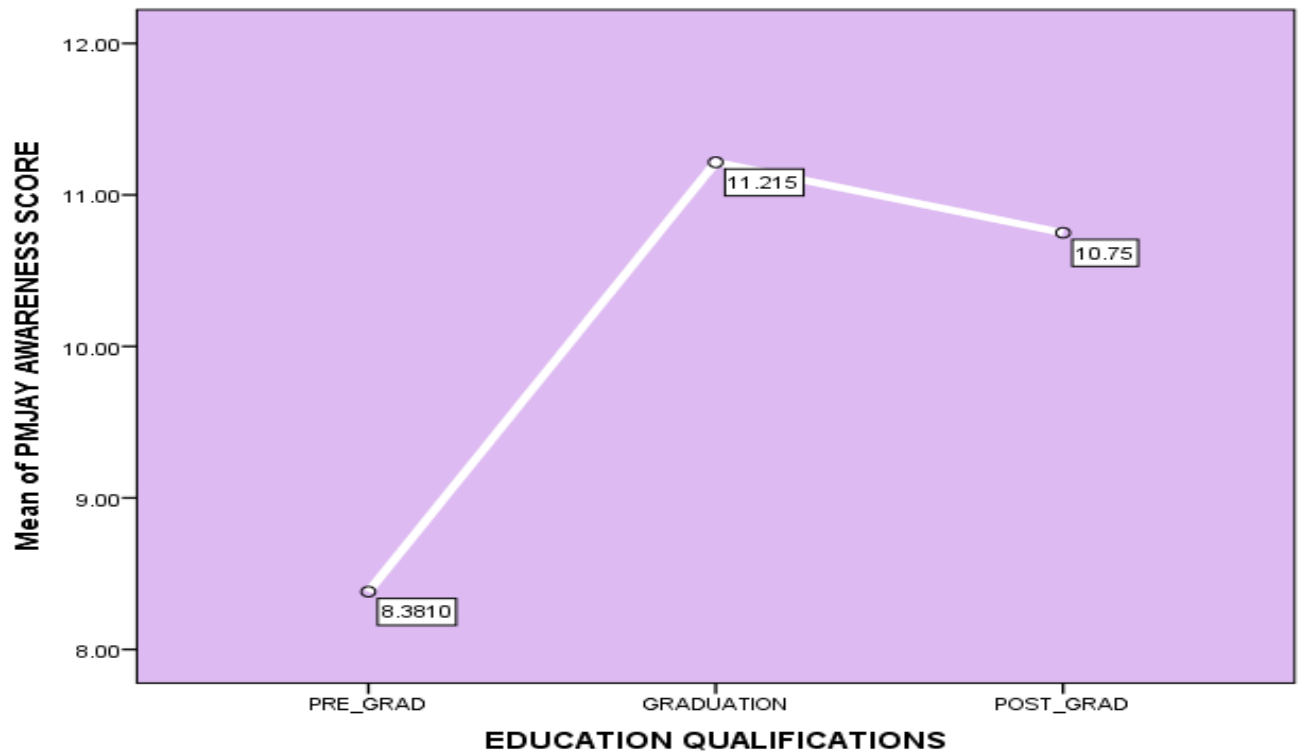

\section{Result \& Interpretation:}

The post hoc test revealed that the Scores of Awareness about PMJAY was statistically significantly different representing lower Awareness score among under graduates $(8.38 \pm 3.73$ marks, $\mathrm{p}=.031)$.

Significant diff. betw ${ }^{\mathrm{n}}$ the Scores of Awareness regarding the Scores of Awareness regarding PMJAY among Graduates $(11.21 \pm 4.49$ marks, $\mathrm{p}=.852)$ and Post graduates $(10.75 \pm 3.98$ marks, $\mathrm{p}=.110)$. Mean plot also suggest the same results.

\section{An effect of Occupation on PMJAY - AB Awareness.}

$\mathrm{H}_{13 \mathrm{~N}}$ : Occupation does not has an effect on PMJAY - AB Awareness.

$\mathrm{H}_{14 \mathrm{~A}}$ : Occupation has an effect on PMJAY - AB Awareness.

To prove that PMJAY Awareness is significantly influenced by different Occupation, test of Analysis of Variance (ANOVA) is conducted.

\begin{tabular}{|l|l|l|l|l|l|}
\hline PMJAY AWARENESS SCORE * OCCUPATION \\
\hline & $\begin{array}{l}\text { Sum_of_ } \\
\text { Squares }\end{array}$ & $\begin{array}{l}\text { Degree_Fr } \\
\text { eedom }\end{array}$ & Mean_Square & F & Sig._(p) \\
\hline Betwn_Grp & 46.480 & 3 & 15.493 & .834 & .478 \\
\hline Within_Grp & 2340.720 & 126 & 18.577 & & \\
\hline Total & 2387.200 & 129 & & & \\
\hline
\end{tabular}




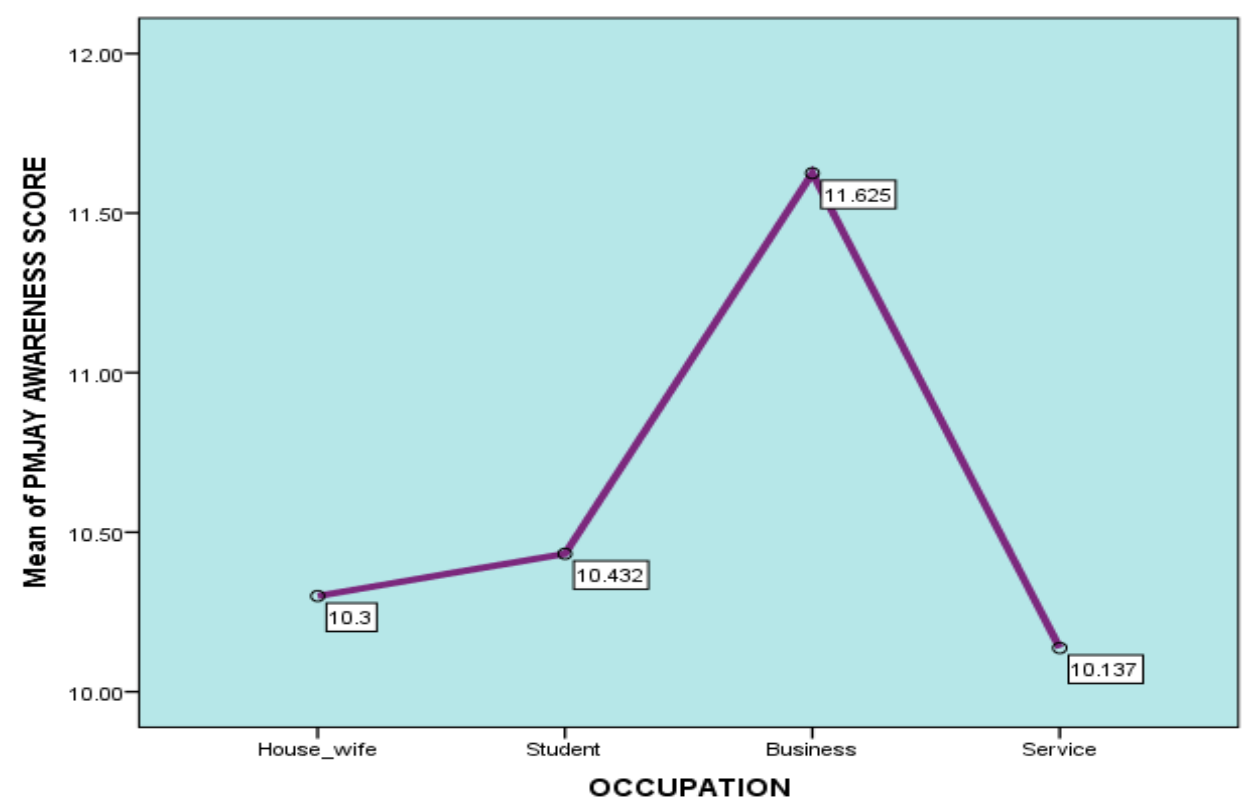

Result \& Interpretation:

There was no significant effect of Occupation on PMJAY Awareness at the $\mathrm{p}=.478>.05$

Post Hoc test cannot apply as there is no significant effect observed.

Null hypothesis is accepted. Same results are observed from mean plot too.

12. An effect of Income on PMJAY - AB Awareness.

$\mathrm{H}_{0}$ : Income does not effect PMJAY - AB Awareness.

$\mathrm{H}_{1}$ : Income effect PMJAY - AB Awareness.

To prove that PMJAY Awareness is significantly influenced by different income status, test of Analysis of Variance (ANOVA) is conducted.

\begin{tabular}{|l|l|l|l|l|l|}
\hline PMJAY AWARENESS SCORE * INCOME & $\begin{array}{l}\text { Sum_of_ } \\
\text { Squares }\end{array}$ & $\begin{array}{l}\text { Degree_Fr } \\
\text { eedom }\end{array}$ & Mean_Square & F & Sig._(p) \\
\hline Betwn_Grp & 91.123 & 4 & 22.781 & 1.240 & .297 \\
\hline Within_Grp & 2296.077 & 125 & 18.369 & & \\
\hline Total & 2387.200 & 129 & & & \\
\hline
\end{tabular}

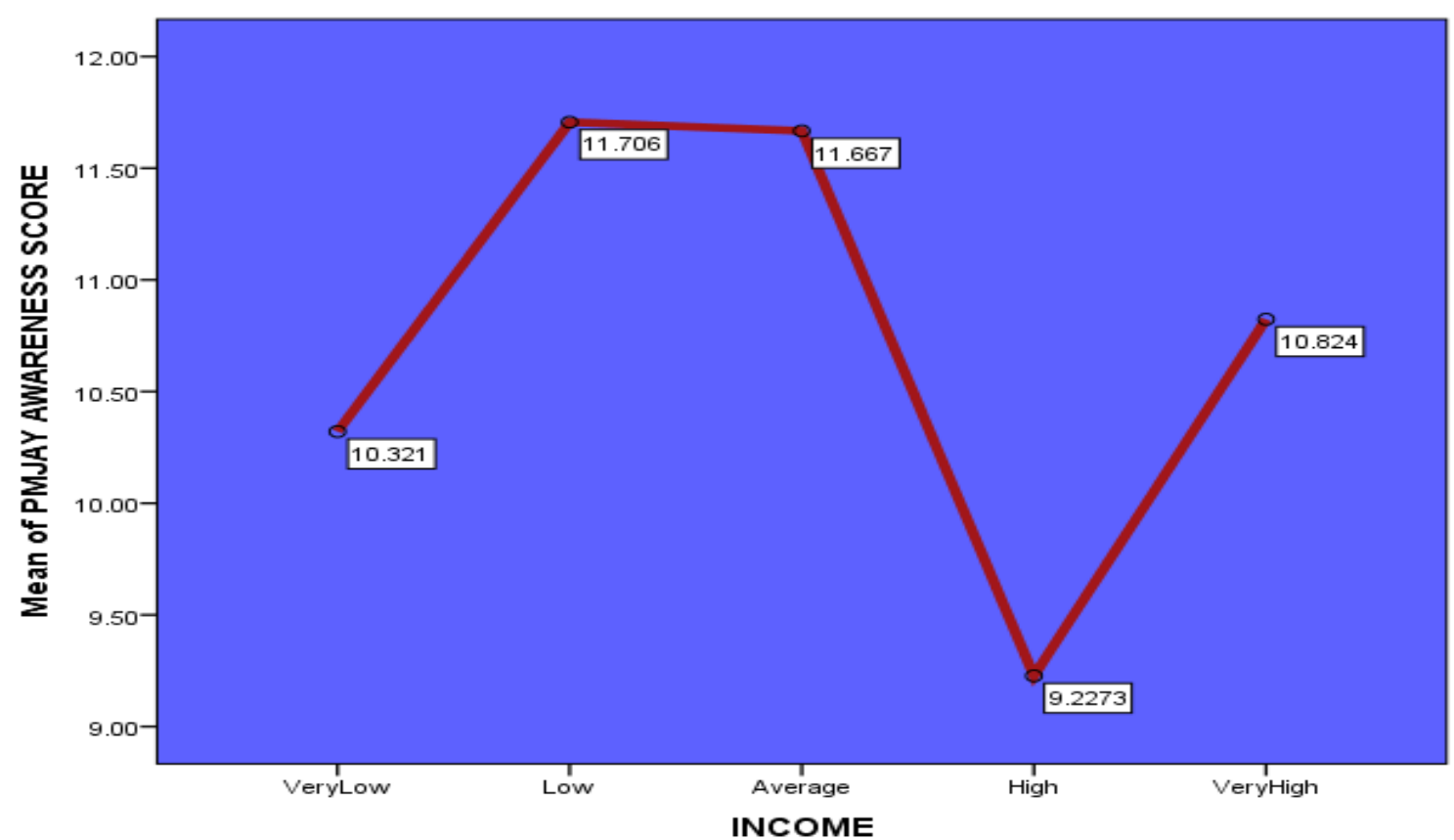

\section{Result \& Interpretation:}

There was no significant effect of Income on PMJAY Awareness at the $p=.297>.05$

Post Hoc test cannot apply as there is no significant effect observed.

Null hypothesis is accepted. Same results are observed from mean plot too. 
An Experimental Evaluation on the awareness of "Pradhan Mantri Jan Aryogya Yojna - Ayushmaan Bharat" (PMJAY-AB) Scheme among residents of Moradabad, Uttar Pradesh

\section{D.Pearson Correlation Test}

13. Association of age and PMJAY-AB Awareness.

$\mathrm{H}_{15 \mathrm{~N}}$ : Awareness regarding PMJAY-AB does not increase with Age.

$\mathrm{H}_{15 \mathrm{~A}}$ : Awareness regarding PMJAY-AB increases with Age.

To find the association between the awareness regarding PMJAY- AB and Age, a Pearson's Correlation coefficient

(r) was calculated:

\begin{tabular}{|l|l|l|l|}
\hline \multicolumn{2}{|l|}{} & AGE & $\begin{array}{l}\text { PMJAY AWARENESS } \\
\text { SCORE }\end{array}$ \\
\hline \multirow{4}{*}{ AGE } & Pearson Correlation (r) & 1 & $.219^{*}$ \\
\cline { 2 - 4 } & Sig. (2-tailed) & & .012 \\
\cline { 2 - 4 } & $\mathrm{N}$ & 130 & 130 \\
\hline \multirow{2}{*}{$\begin{array}{l}\text { PMJAY AWARENESS } \\
\text { SCORE }\end{array}$} & Pearson Correlation (r) & $.219^{*}$ & 1 \\
\cline { 2 - 4 } & Sig. (2-tailed) & .012 & 130 \\
\cline { 2 - 4 } & $\mathrm{N}$ & 130 & \\
\hline *At 0.05_level(2_tailed)_Correlation is found significant & \multicolumn{2}{|l}{} \\
\hline
\end{tabular}

\section{Result \& Interpretation:}

There was a moderate positive correlated-association between the PMJAY-AB awareness and Age, $\mathrm{r}=0.219, \mathrm{n}=$ $130, \mathrm{p}=0.012$

We accept the alternate hypothesis, as results show that with increase in age there is moderately positive increase in awareness regarding PMJAY-AB.

14. Association of income and PMJAY-AB Awareness.

$H_{16 N}$ : Awareness regarding PMJAY-AB does not increase with Income.

$\mathrm{H}_{16 \mathrm{~A}}$ : Awareness regarding PMJAY-AB increases with Income.

To find the association between the awareness regarding PMJAY- AB and Income, a Pearson's Correlation coefficient (r) was calculated:

\begin{tabular}{|l|l|l|l|}
\hline \multicolumn{2}{|l|}{} & $\begin{array}{l}\text { SOCIO } \\
\text { ECONOMIC } \\
\text { STATUS }\end{array}$ & $\begin{array}{l}\text { PMJAY AWARENESS } \\
\text { SCORE }\end{array}$ \\
\hline \multirow{4}{*}{\begin{tabular}{l} 
Income \\
\cline { 2 - 3 }
\end{tabular}} & Pearson Correlation (r) & 1 & -.014 \\
\cline { 2 - 4 } & Sig. (2-tailed) & & .872 \\
\cline { 2 - 4 } $\begin{array}{l}\text { PMJAY AWARENESS } \\
\text { SCORE }\end{array}$ & $\mathrm{N}$ & 130 & 130 \\
\cline { 2 - 4 } & Pearson Correlation (r) & -.014 & 1 \\
\cline { 2 - 4 } & Sig. (2-tailed) & .872 & 130 \\
\hline
\end{tabular}

Result \& Interpretation: There was no correlation between the PMJAY awareness and Income, $\quad \mathrm{r}=-.014, \mathrm{n}=$ $130, \mathrm{p}=0.872$. We accept the null hypothesis, as results show that $\boldsymbol{r}$ is near Val ZERO.

15. Association of Education Qualification and PMJAY-AB Awareness.

$\mathrm{H}_{17 \mathrm{~N}}$ : Awareness regarding PMJAY-AB does not increase with Education qualification.

$\mathrm{H}_{17 \mathrm{~A}}$ : Awareness regarding PMJAY-AB increases with Education qualification.

To find the association between the awareness regarding PMJAY - AB and Education Qualification, a Pearson's Correlation coefficient (r) was calculated:

\begin{tabular}{|c|c|c|c|}
\hline & & \begin{tabular}{|l|} 
EDUCATION \\
QUALIFICATIONS \\
STATUS \\
\end{tabular} & $\begin{array}{l}\text { PMJAY } \\
\text { AWARENESS } \\
\text { SCORE } \\
\end{array}$ \\
\hline \multirow{3}{*}{$\begin{array}{l}\text { EDUCATION } \\
\text { QUALIFICATIONS } \\
\text { STATUS }\end{array}$} & Pearson Correlation (r) & 1 & .139 \\
\hline & Sig. (2-tailed) & & .113 \\
\hline & $\mathrm{N}$ & 130 & 130 \\
\hline \multirow{3}{*}{$\begin{array}{l}\text { PMJAY AWARENESS } \\
\text { SCORE }\end{array}$} & Pearson Correlation (r) & .139 & 1 \\
\hline & Sig. (2-tailed) & .113 & \\
\hline & $\mathrm{N}$ & 130 & 130 \\
\hline
\end{tabular}




\section{Result \& Interpretation:}

There was no correlation between the PMJAY awareness and Education qualification, $\mathrm{r}=.139, \mathrm{n}=130, \mathrm{p}=0.113$

We accept the null hypothesis.

\section{CONCLUSION}

The study concludes that the demographic factors have some relationship, effect and correlation on PMJAY - AB awareness at Moradabad. These demographic factors gender, age, marital status, religions, occupations, socio-economical status were tested with resident's awareness status of PMJAY-AB by conducting descriptive analysis, chi-square test, analysis of variance, post hoc and correlation.

RELATIONSHIP: It is hereby concluded from the results obtained by applying chi-square test (cross tabulation) that except demographic variable Age groups no other variable has a significant relationship with the resident's awareness of PMJAY - AB at Moradabad.

EFFECT: It is hereby concluded from the results obtained by conducting ANOVA that age groups, education level and income-socio-economic level play role in effecting the resident's awareness regarding PMJAY- AB at Moradabad. It is also concluded that very old people (above $60 \mathrm{yrs}$ ) have highest awareness of PMJAY- $\mathrm{AB}$ and young generation (20-30 yrs) have the lowest awareness regarding this government scheme. Further residents between age bands 31-60 yrs have moderate awareness in the Moradabad City. When it comes to education level awareness about PMJAY$\mathrm{AB}$ scheme is least among under graduates and there is no difference between the Awareness of PMJAY- AB among graduates and post graduates in Moradabad.

Other demographic variable does not have any effect on awareness of this government scheme in Moradabad.

Association: It is hereby concluded from the results obtained after applying Pearson Correlation (r) test that except age groups no other variable (education qualification and income) make any association with awareness level of PMJAY- AB scheme at Moradabad.

It is concluded that age has a moderate and positive correlation between the PMJAY - AB awareness at Moradabad.

\section{REFERENCES}

1. Bhattacharjya, A. S., \& Sapra, P. K. (2008). Health insurance in China and India: Segmented roles for public and private financing. Health Affairs.

2. David Mark, D., Radermacher, R., \& Koren, R. (2007). Willingness to pay for health insurance among rural and poor persons: Field evidence from seven micro health insurance units in India. Health Policy, 82(1), 12-27.

3. Devadasan, N.,Criel, B., Van Damme, W., Lefevre, P., Manoharan, S., \& Van der Stuyft, P. (2011). Community health insurance schemes \& patient satisfaction--evidence from India. The Indian Journal of Medical Research, $133,40-49$.

4. Health in India, Social Consumption: Education NSS 71st Round (January2014-June 2014) http://mospi.nic.in/sites/default/files/publication_reports/nss_rep574.pdf

5. Devadasan, N., Criel, B., Van Damme, W.,Manoharan, S., Sarma, P. S., \& Van Der Stuyft, P. (2010). Community health insurance in Gudalur, India, increases access to hospital care. Health Policy and Planning, 25(2), $145-154$.

6. Devadasan, N., Ranson, K., Damme, W. Van, \& Criel, B. (2004). Community Health Insurance in India: An Overview. Economic and Political Weekly, 39(28), 3179-3183. doi:10.2307/4415264

7. Devadasan, N., Ranson, K., Van Damme, W., Acharya, A., \& Criel, B. (2006). The landscape of community health insurance in India: An overview based on 10 case studies. Health Policy, 78(2-3), 224-234
8. Ito, S., \& Kono, H. (2010). Why is the take-up of micro insurance so low? Evidence from a health insurance scheme in India. Developing Economies, 48(1), 74-101. 